\title{
RELAXATION EFFECTS ON THE FLOW OVER SLENDER BODIES
}




\section{THE COLLEGE OF AERONAUTICS}

\section{CRANFIELD}

\section{Relaxation Effects on the Flow over Slender Bodies \\ - by -}

J. F. Clarke, B.Sc., Ph.D.

A. F.R.A.S.

Department of Aerodynamics

\section{SUMIMARY}

The effects of heat capacity lag on the flow over slender bodies are examined via an extension of Ward's (1949) generalised treatment of the slender body problem. The results are valid for smooth bodies of arbitrary cross-sectional shape and attitude in the complete Mach number range up to, but not including, hypersonic. Transonic flow can be treated owing to the presence of a dissipative mechanism in the basic differential equation, but the results in this Mach number range are probably of limited practical value.

The resulis show that cross-wind forces are unaffected in a first approximation, but that drag forces comparable with laminar skin friction values can arise as a result of the relaxaticn of the internal degrees of freedom. The magnitude and sign of these effects depend strongly on body shape and free stream Miach number.

Results are given for surface pressure coefficient and the variations of translational and internal mode temperature on and near the body are also found. The influence of these latter effects on heat transfer to the body is discussed. 


\section{CONTENTS}

Page

Summary

List of Symbols

1. Introduction 1

2. The Equations 3

3. The General Solution 10

4. The Values of $b_{0}(x) \quad 14$

5. Drag 19

6. The Pressure Coefficient 32

7. The Temperature Variations 35

8. References 39

Appendix A - Inversion integrals for the function $b_{0}(x) \quad 41$

Apperrix B - Evaluation of the relaxation drag integral 


\section{LIST OF SYMBOLS}

$B$ defined in equation 40

$\mathrm{B}_{\mathrm{e}} \quad$ defined in equation 40

$B_{f} \quad$ defined in equation 40

$\mathrm{C}_{\mathrm{D}} \quad$ drag coefficient

$\mathrm{C}_{\mathrm{p}} \quad$ specific heat at constant pressure

$\mathrm{C}_{\mathrm{v}} \quad$ specific heat at constant volume

D drag

e internal energy per unit mass

h enthalpy per unit mass

L body length

MI Mach number

p pressure

9 velocity vector

$\mathrm{R}$ gas constant

s entropy per unit mass

$\mathrm{S} \quad$ body cross-section area

$\mathrm{S}_{r} \quad$ reference area

$\mathrm{T}$ temperature

$\mathrm{u}, \mathrm{v}, \mathrm{w}$ velocity components

$\mathrm{U}$ free stream velocity

$\mathrm{x}, \mathrm{r}, \theta$ cylindrical polar co-ordinates

$\beta \quad$ defined in equation 34

$\bar{\beta} \quad$ defined in equation 37

$\beta$ e defined in equation 34

$\beta_{f} \quad$ defined in equation 34

$\lambda$ relaxacion length

$\rho$ density

$\tau^{\prime}$ relaxation time

$\tau$

effective relaxation time (Equation 18) 
List of Symbols (Continued)

$\phi \quad$ velocity potential

$\Phi$ Fourier transform of $\phi$

$\omega \quad$ transform variable

\section{Suffixes}

translational plus "active" degrees of freedom relaxing internal mode

$\infty \quad$ free stream conditions

e equilibrium

f frozen 


\section{Introduction}

In the sections to follow we shall examine the flow of a polyatomic gas about slender three-dimensional bcdies, taking into account the effects of heat capacity lag. To simplify the problem we shall assume that the gas is inviscid and non-heat conducting, although we shall attempt, very briefly, to indicate how these gas properties fit into the simpler flow patterns obtained here.

From a practical point of view, relaxation effects are likely to assume greater significance as the general leve? of gas pressure decreases. The relaxation times for adjustment of internal molecular states to conditions of thermal equilibrium vary inversely with pressure and it is conceivable that we may find "relaxation lengths" comparable with overall body length in some circumstances. ("Relaxation length" is the product of relaxation time $\tau$ and free stream speed $U)$. Provided that the number of collisions required to excite the internal mode is sufficiently large, it is possible to encounter such conditions within the regime of continuum flow. We treat the problem on the basis of this possibility.

The analysis to follow makes no explicit reference to any particular gas or mixture of gases, but some examples are given for $\mathrm{CO}_{2}$, a gas which exhibits some of the required effects in circumstances which are practically realizable.

The effects of internal mode relaxations on gas dynamic behaviour have been investigated previously by Gunn (1952), who gives an account of sound absorption and dispersion, shock wave effects, one-dimensional nozzle flows and of the drag experienced by an object as a result of the dissipative actions of relaxation (the latter confined to two-dimensions). The drag problems treated by Gunn are generally tackled by finding the perturbations introduced into a "non-relaxing" gas flcw and he does not 
consider questions of transonic or supersonic flow. Here we attempt to "unify" the treatment in the manner of Ward's (1949) solution of the inert gas slender body problem. The general question of relaxing and reacting gas flows has received a certain amount of attention recently and it is pertinent to remark here on the formal similarity which exists between heat capacity lag effects and those which arise when endothermic chemical reactions occur in a gas flow. This similarity is made apparent by the fundamental work of Kirkwood and Wood (1957) and appears also in recent papers by Moore and Gibson (1960) and Vincenti (1960). An analysis of the supersonic flow of a chemically reacting gas round a sharp corner which would apply equally well to a relaxation effect problem has been given by Clarke (1960a). Moore and Gibson base their considerations on a velocity potential which satisfies the telegraph equation. The latter can be shown to approximate to the "exact" small perturbation equation for cases where the differences between frozen and equilibrium sound speeds are small. Vincenti considers the "wavy wall" problem, thereby considering a type of fundamental solution of the steady two-dimensional small perturbation equation for reacting or relaxing gas flows, using, as does Clarke, the "exact" small disturbance equation. The one-dimensional unsteady analogue of Vincenti's problem (the harmonically oscillating piston) has been examined by Clarke (1958) and the step-input piston problem in a reacting gas has been treated by Chu (1957).

Most of the work to date, therefore, has been concerned with "fundamental" types of solutions, or "inputs", to the gas. One may perhaps level some criticism, from a practical point of view, at the "wavy wall" and harmonically oscilleting piston problem solutions, since it is an essential feature of relaxation or reaction effects that entropy shall be produced continuously during the time taken to reach a new equilibrium state. The infinite past histories of these processes 
would therefore, strictly, require an infinite difference of entropy between the postulated "undisturbed" regions and those adjacent to the object. Such (rather pedantic) objections are removed when the disturbing influence is of finite physical size (or duration).

As noted by Vincenti, the inclusion of a dissipative mechanism into the analysis permits one to obtain continuous solutions from a purely linear equation right through the "transonic" region. Indeed, the presence of an infinity of sound speeds, ranging from the frozen to full equilibrium values, smears the transonic region over a finite band of free strecim velocities, so that the singular behaviour of the linear flow equations is confined to only one frequency of the infinite range which must be superimposed to summarise the effect of the obstacle as a whole.

\section{The Equations}

We shall assume in $v$ hat follows that only one internal energy mode exhibits significant relaxation effects. Any other modes are treated as "active" modes and it is supposed that their energy content is specified once the translational temperature, $\mathrm{T}_{1}$, is known. Following Kirkwood and Wood (1957) it is assumed that the state of the relaxing mode is described by another temperature, $T_{2}$, which only equals $T_{1}$ when complete thermal equilibrium prevails. The specific internal energy of the gas, e, is made up as follows :

$$
e=e_{1}+e_{2}
$$

where

$$
\begin{aligned}
& e_{1}=\int_{0}^{T_{1}} C_{v_{1}} d T_{1}, \\
& e_{2}=\int_{0}^{T_{2}} C_{v_{2}} d T_{2} .
\end{aligned}
$$


$\mathrm{C}_{\mathrm{V}_{1}}$ and $\mathrm{C}_{\mathrm{V}_{2}}$ are the specific heats at constant volume for the translational plus active degrees of freedom of the molecule and the relaxing mode, respectively. When $e_{2}$ is in equilibrium with $e_{1}$ the upper limit of integration in equation 3 is replaced by $T_{1}$. We write $C_{V}$ for the total specific heat at constant volume i.e. :

$$
\mathrm{C}_{\mathrm{v}}=\mathrm{C}_{\mathrm{v}_{1}}+\mathrm{C}_{\mathrm{v}_{2}}
$$

(where $\mathrm{C}_{\mathrm{V}_{1}}$ and $\mathrm{C}_{\mathrm{V}_{2}}$ are temperature dependent equation 4 only has a meaning when $T_{i}=T_{2}$ ). Note that a similar notation is followed with specific heats at constant pressure. We shall write

$$
\mathrm{C}_{\mathrm{p}}=\mathrm{C}_{\mathrm{p}_{1}}+\mathrm{C}_{\mathrm{v}_{2}} \text {. }
$$

The pressure $p$, density $\rho$ and translational temperature are related via

$$
\mathrm{p}=\rho \mathrm{R} \mathrm{T}_{1}
$$

where $R$ is the appropriate gas constant per unit mass. The gas is therefore treated as thermally perfect.

The relaxation of the internal mode to an equilibrium state is assumed to be described by the linear law

$$
\tau^{\prime} \frac{\operatorname{De}_{2}\left(T_{2}\right)}{D t}=e_{2}\left(T_{1}\right)-e_{2}\left(T_{2}\right)
$$

$\tau^{\prime}$ is therefore the relaxation time, $D / D t$ the usual convective operator and $e_{2}\left(T_{1}\right)$ signifies that the internal mode has an energy content appropriate to the actual, local, translational temperature. Since we are to deal with small perturbations from an originally undisturbed (equilibrium) stream it is reasonable to assume that $\tau^{\prime}$ is constant throughout. Furthermore, since $T_{2}$ will not differ greatly from $T_{1}$ in these circumstances, equation 3 shows that we can approximate to equation 7 by

$$
\tau^{\prime} \frac{\mathrm{DT}_{2}}{\mathrm{Dt}}=\mathrm{T}_{1}-\mathrm{T}_{2} \text {. }
$$


This is tantamount to saying that $\mathrm{C}_{\mathrm{V}_{2}}$ is a constant, evaluated at the free stream temperature $T_{\infty}$ and, in the small disturbance problem, we may say likewise for $\mathrm{C}_{\mathrm{V} 1}$.

The energy equation can be written as

$$
\mathrm{C}_{\mathrm{V}_{1}} \frac{\mathrm{DT}}{\mathrm{Dt}}+\mathrm{C}_{\mathrm{V}_{2}} \frac{\mathrm{DT} \mathrm{T}_{2}}{\mathrm{Dt}}+\frac{\mathrm{p}}{\rho} \operatorname{div} \mathrm{q}=0
$$

and $\mathrm{C}_{\mathrm{V}_{1}}$ and $\mathrm{C}_{\mathrm{V}_{2}}$ can subsequently be treated as constants. $\mathrm{g}$ is the gas velocity vector and we do not introduce any further linearisation at the present stage. $\mathrm{DT}_{1} / \mathrm{Dt}$ can be eliminated from equation 9 via equation 8 , giving

$$
\mathrm{C}_{\mathrm{V}} \frac{\mathrm{DT}}{\mathrm{Dt}}+\tau^{\prime} \mathrm{C}_{\mathrm{V}_{1}} \frac{\mathrm{D}}{\mathrm{Dt}}\left(\frac{\mathrm{DT}_{2}}{\mathrm{Dt}}\right)+\frac{\mathrm{p}}{\rho} \operatorname{div} \underset{\sim}{\mathrm{q}}=0 .
$$

From the results of Kirkwood and Wood it is found that the mass conservation requirement can be written in terms of pressure rather than density derivatives, giving

$$
\frac{D p}{D t}+\rho a_{1}^{2} \operatorname{divq}+\rho a_{1}^{2}\left(\beta \beta_{1} / C_{p_{1}}\right) \frac{D e_{2}}{D t}=0 .
$$

This equation introduces the frozen sound speed $a_{1}$ and we readily find that

$$
a_{1}^{2}=\left(C_{p_{1}} / C_{v 1}\right)(p / \rho)
$$

$\beta_{1}$ is the volume expansion coefficient for variations of translational temperature only, i.e.

$$
\beta_{1}=\left(\frac{\partial \rho^{-1}}{\partial T_{1}}\right)_{p}=\left(\rho T_{1}\right)^{-1} .
$$

Using equations 12 and 13 in equation 11 , it follows that, in the present case,

$$
\frac{D p}{D t}+\rho a_{1}^{2} d i v \underset{\sim}{q}+\rho R\left(C_{V_{2}} / C_{V_{1}}\right) \frac{D_{2}}{D t}=0
$$


Writing the equilibrium speed of sound as $a_{2}$, where

$$
a_{2}^{2}=\left(C_{p} / C_{v}\right)(p / p)
$$

it quickly follows from equations 10 and 14 that

$$
\left(\mathrm{C}_{\mathrm{v}_{1}} \tau^{\prime} / \mathrm{C}_{\mathrm{v}}\right) \frac{\mathrm{D}}{\mathrm{Dt}}\left\{\frac{1}{\rho} \frac{\mathrm{Dp}}{\mathrm{Dt}}+\mathrm{a}_{1}^{2} \operatorname{div} \underset{\sim}{\mathrm{q}}\right\}+\frac{1}{\rho} \frac{\mathrm{Dp}}{\mathrm{Dt}}+\mathrm{a}_{2}^{2} \operatorname{div} \underset{\sim}{\mathrm{q}}=0 .
$$

Now we would anticipate, on physical grounds, that the presence of relaxation effects in a gas flow will serve to make "detailed" changes in the flow pattern when compared with a corresponding inert gas problem, but will not change the "orders of magnitude" of quantities involved. For example, in the case of the reacting gas flow round a sharp corner, the pressure coefficient on the surface is found to vary from $-2 \theta / \mathrm{B}_{\mathrm{f}}$ to $-2 \theta / B_{e}$ where is the turaing angle and $B_{f}$ and $B_{e}$ are the usual Ackeret Mach number factors based on the frozen and equilibrium sound speeds, respectively. The variation from a $B_{f}$ to a $B_{e}$ type of factor represents a "detailed" change of pressure coefficient, but its "order of magnitude" remains at the value $\theta$. There is no reason to suspect that this situation will alter when we come to consider the three-dimensional situation involved in the present slender pointed body problem and, accordingly, we accept, as a general guide, the orders of magnitude given in Ward's (1949) paper. It should be remarked at this stage that we are only going to deal with steady flow problems in what follows. Henceforth, therefore, the operator D/Dt becomes synonymous with q grad. Thus we siall follow the rigorously justifiable procedure in the case of inert gas flow past slender bodies (see e.g. Lighthill, 1945) of linearising the basic differential equation but keeping appropriate non-linear terms in the relation between pressure and velocity.

The pointed nose of the body is assumed to lie at the origin of a "wind-axes" co-ordinate system. The free stream velocity will be written as $U$ in the direction of the Ox axis. 
In order to linearise equation 16 then, we first replace the convective

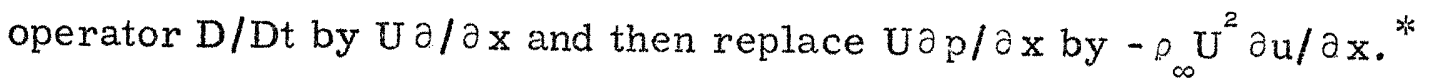
The resulting equation will be written in a cylindrical polar co-ordinate system $(x, r, \theta)$ in which the disturbance velocity components have the values $u, v, w$ respectively. In addition of course, all terms multiplying the derivatives of disturbance velocities will be given their free stream ( $\operatorname{suffix} \infty$ ) values. Writing out the divergence term in full, the result of these linearisations is

where

$$
\begin{aligned}
\tau U \frac{\partial}{\partial x}\left\{\left(1-M_{f}^{2}\right) \frac{\partial u}{\partial x}+\frac{1}{r} \frac{\partial(r v)}{\partial r}+\frac{1}{r} \frac{\partial w}{\partial \theta}\right\} \\
+\left(1-M_{e}^{2}\right) \frac{\partial u}{\partial x}+\frac{1}{r} \frac{\partial(r v)}{\partial r}+\frac{1}{r} \frac{\partial w}{\partial \theta}=0,
\end{aligned}
$$

$$
\begin{aligned}
\tau & =\mathrm{C}_{\mathrm{p}_{1}} \tau^{\prime} / \mathrm{C}_{\mathrm{p}}, \\
\mathrm{M}_{\mathrm{f}} & =\mathrm{U} / \mathrm{a}_{1 \infty} ; \quad \mathrm{MI}_{\mathrm{e}}=\mathrm{U} / \mathrm{a}_{2 \infty} .
\end{aligned}
$$

$\mathrm{M}_{\mathrm{f}}$ and $\mathrm{MI}_{\mathrm{e}}$ are the frozen and equilibrium Mach numbers respectively.

*

This result comes from the linearised version of the $\mathrm{x}$-wise momentum. equation. It is acceptable in deriving equation 17 (but not in deriving in the pressure/velocity relation) provided that we can neglect vorticity. Justification for neglecting vorticity is discussed below. 
The thermodynamic equation for a relaxing gas of the type considered here is

$$
T_{1} d s=d h-\rho^{-1} d p+\left(T_{1}-T_{2}\right) T_{1}^{-1} d e_{2}
$$

(Kirkwood and Wood, loc. cit.) where $h$ is the specific enthalpy,

$$
\mathrm{h}=\mathrm{e}+\mathrm{p} / \mathrm{\rho}
$$

and $\mathrm{s}$ is the specific entropy. When the energy equation is written in the form

$$
\frac{\mathrm{Dh}}{\mathrm{Dt}}-\frac{1}{\rho} \frac{\mathrm{Dp}}{\mathrm{Dt}}=0
$$

it is at once apparent that

$$
T_{1} \frac{D s}{D t}=\left(T_{1}-T_{2}\right) T_{1}^{-1} \frac{D e_{2}}{D t}=C_{V 2}\left(T_{1}-T_{2}\right)^{2} / T_{1} T^{i}
$$

(using equations 3 and 8 ). The rate of entropy rise of a fluid particle is thus of second order in the difference between $T_{1}$ and $T_{2}$.

In the steady state, the exact momentum equations can be written as

$$
\operatorname{grad}\left(q^{2} / 2\right)+\operatorname{grad} p-\rho \underset{\sim}{q} x \operatorname{curl} \underset{\sim}{q}=0
$$

whence it follows irom equation 20 that

$$
-q^{x} \operatorname{curl} g=T_{1} \operatorname{grads}+T_{1}^{-1}\left(T_{2}-T_{1}\right) \operatorname{grad} e_{2}-\operatorname{grad} h{ }_{0},
$$

where $h_{0}=h+q^{2} / 2$ is the stagnation euthaipy. We can show that, in steady flow, $h_{0}=$ const. on streamlines, whence, since the flow is assumed to originate in a region of uniform $h_{0}$, grad $h_{0}=0$ everywhere.

It is a little difficult to generalise about the behaviour of the terms involving grads and grade ${ }_{2}$ in equation 25 , since their actual magnitude will depend on the body shape as well as on relaxation time etc. The majority of the resuits in the present paper will be obtained on the assumption that curl $\underset{\sim}{q}$ can be neglected in a first (linear) approximation. To justify this to some extent we observe that $T_{1} D s / D t$ and $\left(T_{1}-T_{2}\right) T_{1}^{-1} \mathrm{De} / \mathrm{Dt}$ 
are equal (see equation 23). In equation 101 below a general result for the difference $T_{1}-T_{2}$ is obtained. In supersonic flow the lower limit in the integral in equation $101 \mathrm{is} \mathrm{replaced} \mathrm{by} \mathrm{zero.} \mathrm{Then}\left(\mathrm{T}_{1}-\mathrm{T}_{2}\right) \mathrm{T}_{1}^{-1}$ is of order $M_{f}^{2}$ (C.P. $)_{m}^{\prime} \lambda\left(1-e^{-x / \lambda}\right)$, where (C.P. $)_{m}^{\prime}$ is a mean value of pressure coefficient gradient between 0 and $x$. It follows that the gradien $i$ of $s$ (for example) along a streamline is of order $\mathbb{M}_{f}^{4}$ (C.P. $)_{m}^{12}$ $\lambda\left(1-\mathrm{e}^{-\mathrm{x} / \lambda^{2}{ }^{2} \mathrm{C}} \mathrm{V}_{2}\right.$. Now the pressure coefficient is of order body thickness ratio squared and hence so will be (C.P. $)_{m}^{\prime}$, roughly speaking. The appearance of the square of (C.P. $)_{m}^{\prime}$ certainly suggests that even g:ads will be small for "reasonable" slender shapes and the neglect of curl $\underset{\sim}{\mathrm{q}}$ would seem to be justified. Due to the singular behaviour of C.P. at $x=0$ in subsonic or transonic flow (see below) it is not quite so easy to estimate how the integral in equation 101 behaves. However, on physical grounds, there seems no reason to doubt that what will apply to supersonic flow will also hold in these flow regimes. This being so, in the linear approximation we may define a disturbance velocity potential $\phi$ where

$$
u=\frac{\partial \phi}{\partial x}: v=\frac{\partial \phi}{\partial r}: w=\frac{1}{r} \frac{\partial \phi}{\partial \theta} .
$$

In these terms, equation 17 becomes

$$
\begin{aligned}
\lambda \frac{\partial}{\partial x}\left\{\left(1-\mathbb{M}_{f}^{2}\right) \frac{\partial^{2} \phi}{\partial x^{2}}+\frac{1}{r} \frac{\partial}{\partial r}\left(r \frac{\partial \phi}{\partial r}\right)+\frac{1}{r} \frac{\partial^{2} \phi}{\partial \phi^{2}}\right\} & \\
& +\left(1-\mathbb{M}_{e}^{2}\right) \frac{\partial^{2} \phi}{\partial x^{2}}+\frac{1}{r} \frac{\partial}{\partial r}\left(r \frac{\partial \phi}{\partial r}\right)+\frac{1}{r^{2}} \frac{\partial^{2} \phi}{\partial \theta^{2}}=0,
\end{aligned}
$$

where we have written

$$
\tau U=\lambda \text {. }
$$

$\lambda$ is the characteristic relaxation length.

Accepting the linear theory approximation curl $\underset{\sim}{q} \simeq 0$, it follows from equation 29 that a proper expression for the pressure coefficient is

(see Ward, 1949).

$$
(\text { C.P. })=2\left(p-p_{\infty}\right) / \rho_{\infty} U^{2} \bumpeq-\frac{2}{U} \frac{\partial \phi}{\partial x}-\frac{1}{U^{2}}\left(\frac{\partial \phi}{\partial r}\right)^{2}-\frac{1}{U^{2}} \frac{1}{r^{2}}\left(\frac{\partial \phi}{\partial \theta}\right)^{2},
$$




\section{The General Solution}

The general solution of equation 27 can most easily be obtained by using the exponential Fourier transform. Thus we define

$$
\Phi=\frac{1}{\sqrt{2 \pi}} \int_{-\infty}^{\infty} \phi e^{i \omega x} d x .
$$

Assuming that $\phi, \partial \phi / \partial x$ and $\partial^{2} \phi / \partial x^{2}$ all vanish with increasing distance from the body, it is found that $\Phi$ satisfies the following differential equation.

$$
\begin{aligned}
\frac{\partial^{2} \Phi}{\partial r^{2}} & +\frac{1}{r} \frac{\partial \Phi}{\partial r}+\frac{1}{r^{2}} \frac{\partial^{2} \Phi}{\partial \theta^{2}} \\
& -\omega^{2}\left(1-M_{f}^{2}\right)\left[\frac{1-M_{e}^{2}}{1-M_{f}^{2}}-i \omega \lambda\right][1-i \omega \lambda]^{-1} \Phi=0 .
\end{aligned}
$$

Expressing $\Phi$ as a Fourier series in $\theta$ we find that each coefficient of $\cos n \theta$ or $\sin n \theta$ is the solution of a modified Bessel equation, of order $\mathrm{n}$. In fact we can write the solution formally as

$$
\Phi(r, \theta: \omega)=A_{0}(\omega) K_{0}\left(r \omega \sqrt{1-M_{f}^{2}} z\right)+\sum_{n=1}^{\infty} A_{n}(\omega) K_{n}\left(r \omega \sqrt{1-M_{f}^{2}} z\right) \cos \left(n \theta+\varepsilon_{n}\right)
$$$$
\ldots \ldots
$$

where $A_{n}, n=0,1,2, \ldots$ and $\varepsilon_{n}, n=1,2, \ldots$ are "constants" which are to be evaluated from the boundary corditions on the body. Also,

$$
Z=\left\{\omega+i \lambda^{-1}\left(\frac{1-M_{e}^{2}}{1-M_{f}^{2}}\right)\right\}^{\frac{1}{2}}\left[\omega+i \lambda^{-1}\right]^{-\frac{1}{2}} \text {. }
$$

The $I_{n}-t_{y}$ pe functions are rejected in the solution for $\Phi$ because they are found not to represent the proper type of "outgoing" wave system.

In interpreting equation 32 we must distinguish between the three following cases. 
(i) $\mathrm{M}_{\mathrm{f}}<\mathrm{M}_{\mathrm{e}}<1 ;$ the subsonic case

Here we shall write

$$
\sqrt{1-M_{f}^{2}} \quad=\beta_{f}:\left(1-M_{e}^{2}\right) /\left(1-M_{f}^{2}\right)=\beta^{2}(<1) .
$$

In this case we must write the argument of the Bessel functions as

$$
r \beta_{f}|\omega| z
$$

where

$$
z=\left(\omega+i \lambda^{-1} \beta^{2}\right)^{\frac{1}{2}}\left(\omega+i \lambda^{-1}\right)^{-\frac{1}{2}},
$$

and invert the transform along the reai $\omega$-axis.

(ii) $\mathbb{M}_{f}<1<\mathbb{M}_{\mathrm{e}}$; the transonic case

$$
\sqrt{1-\mathbb{N}_{f}^{2}}=\beta_{f}:\left(M_{e}^{2}-1\right) /\left(1-\mathbb{M}_{f}^{2}\right)=\bar{\beta}^{2}
$$

Note that $0<\bar{\beta}^{2}<\infty$. The proper behaviour of the solution is then assured if inversion takes place on a contour indented to pass above the singularity at $\omega=0$, and $b \in$ ginning and ending at $\omega=\infty \mathrm{e}^{\mathrm{i} \pi}$ and $+\infty$ respectively.

The argument of the Bessel functions is now written as

$$
r \beta_{f} \omega Z^{\prime}
$$

where

$$
z^{\prime}=\left\{\omega-i \lambda^{-1} \bar{\beta}^{2}\right\}^{\frac{1}{2}}\left\{\omega+i \lambda^{-1}\right\}^{-\frac{1}{2}} .
$$

(iii) $M_{e}>M_{f}>1$; the supersonic case

When these Mach number conditions are satisfied we write

$$
\left.\sqrt{M_{f}^{2}-1}=B_{f}:\left(M_{e}^{2}-1\right) /\left(M_{f}^{2}-1\right)=B^{2}:>1\right) .
$$

The argument of the Bessel functions is now written as

where

$$
\mathrm{e}^{\mathrm{i} \pi / 2} \mathrm{r} \omega \mathrm{B}_{\mathrm{f}} z^{\prime \prime}
$$

$$
z^{\prime \prime}=\left\{\omega+i B^{2} \lambda^{-1}\right\}^{\frac{1}{2}}\left\{\omega+i \lambda^{-1}\right\}^{-\frac{1}{2}} \text {. }
$$


Proper behaviour of the solution is guarant eed if we use the inversion contour described in Case (ii).

In dealing with slender bodies we can now approximate to equation 32 near the body by retaining only the first terms in the series expansions of the Bessel functions. Then it is found that

$$
\begin{aligned}
\Phi(r, \theta: \omega) & \simeq-A_{0}\left\{C+\log \left[\left(r\left|1-M_{f}^{2}\right|^{\frac{1}{2}} \omega z\right) / 2\right]\right\} \\
& +\frac{1}{2} \sum_{n=1}^{\infty}(n-1) ! A_{n} 2^{n}\left(r\left|1-M_{f}^{2}\right|^{\frac{1}{2}} \omega Z\right)^{-n} \cos \left(n \theta+\varepsilon_{n}\right)
\end{aligned}
$$

where $\mathrm{C}$ is Euler's constant, $=0.5772 \ldots$ The terms written formally as $\omega Z$ in equation 43 must be interpreted in the forms given above in each case (i), (ii) and (iii). Following Ward we can now define the complex variable $\zeta$ in cross-flow planes, i.e.

$$
\zeta=r e^{i \theta}
$$

whence equation 43 is equivalent to

$$
\Omega=\phi+i \psi=a_{0}(x) \log \zeta+b_{0}(x)+\sum_{n=1}^{\infty} a_{n}(x) \zeta^{-n} .
$$

The quantities $a_{0}, b_{0}$ and $a_{n}, n=1,2,3, \ldots$ are defined as follows (using the symbol " $\supset$ " to mean "has the Fourier transform").

$$
\begin{gathered}
a_{0}(x) \Rightarrow-A_{0}(\omega): b_{0}(x) \Rightarrow-A_{0}(\omega)\left[C+\log \left[\left(1-\left.M_{f}^{2}\right|^{\frac{1}{2}} \omega Z\right) / 2\right]\right\}: \\
a_{n}(x) \supset \frac{1}{2}(n-1) ! A_{n}(\omega) 2^{n}\left(\left|1-M_{f}^{2}\right|^{\frac{1}{2}} \omega z\right)^{-n} e^{i^{E}} .
\end{gathered}
$$

The quantity $\Omega-b_{0}$, is the same harmonic function in both this case and in Ward's, implying in the present case that the "incompressible cross-How" approximation is still valid. In other words, the $a_{n}(x)$ quantities $(\mathrm{n} \geqslant 1)$ can still be found by rolving a two-dimensional, incompressible, pctential flow problem with suitable boundary conditions imposed on the surface of a cylinder whose cross-section is that of the body at any chosen station $x$. To the present order of approximation, 
these $a_{n}(x)$ terms are therefore unaffected by relaxation effects.

Since Ward's momentum integral results for overall forces on the body apply equally well here, it is concluded that both the overall and local cross-flow forces are unaffected by relaxation in the linear approximation.

Omitting the base drag term where blunt-based bodies are concerned, the inert gas flow expression for drag (which is equally applicable here) is

$$
\mathrm{D} / \frac{1}{2} \rho_{\infty} \mathrm{U}^{2}=4 \pi \int_{0}^{\mathrm{L}} \frac{\mathrm{a}_{0}^{\prime}}{\mathrm{U}} \cdot \frac{\mathrm{b}_{\mathrm{o}}}{\mathrm{U}} \mathrm{dx}-2 \pi\left(\frac{\mathrm{o}_{\mathrm{O}} \mathrm{b}_{\mathrm{o}}}{\mathrm{U}^{2}}\right)_{\mathrm{x}=\mathrm{L}}-\frac{1}{\mathrm{U}^{2}}\left(\int_{\mathrm{C}} \phi \frac{\partial \phi}{\partial v} \mathrm{~d} \sigma\right)_{\mathrm{x}=\mathrm{L}} .
$$

In ecuation 46 we have written $a_{0}^{l}$ for the $x$-derivative of $a_{0} L$ is the body length along the $x$-axis and the (pointed) nose of the body lies at $x=0$. In the final integral, $\partial \phi / \partial v$ represents the disturbance potential derivative taken along the outwards normal to the body crosssection contour and the integral is taken around the perimeter of this cross-section.

As before,

$$
a_{0}(x)=U \frac{S^{\prime}(x)}{2 \pi},
$$

where $S(x)$ is the body's cross-sectional area. The prime on $S$ represents differentiation with respect to $x$. Clearly $S^{\prime}(0)=0$ for any streamlined body which begins at $x=0$, since the body radius is zero there by definition. The additional restriction to nointed nosed bodies, mentioned above, is necessary for the linearisations of the basic equations to be valid. Only in this case will the disturbance velocities remain small compared with $U$ over most of the region adjacent to the body.

The presence of upstream influence effects in the sub and transonic regimes, implies that we should confine our attention to bodies which 
are also pointed at the tail $(x=L)$ in these cases, at least in the absence of a priori knowledge about viscous wake patterns. With this additional restriction, equation 46 reduces to

$$
\mathrm{D} / \frac{1}{2} \rho_{\infty} \mathrm{U}^{2}=4 \pi \int_{0}^{L} \frac{\mathrm{a}_{0}^{l}}{\mathrm{U}} \frac{\mathrm{b}_{0}}{\mathrm{U}} \mathrm{dx} \text {. }
$$

Equation 46 will only be used in full in the supersonic regime, where the absence of upstream influence permits us to deal with blunt-based bodies.

\section{The Values of $b_{0}(x)$}

The solution of the relaxation problem is completed once $b_{0}$ is found as a function of $x$. In other words we must invert the Fourier transforms given in equation 45 , taking care to treat each case, (i), (ii) and (iii) above separately. The actual evaluations are carried out in Appendix A and yield the following results.

$$
\begin{aligned}
& \text { Case (i) } \\
& \begin{aligned}
2 \pi \frac{b_{o}}{U} & =S^{\prime}(x) \log \left(\beta_{f} / 2\right)-\frac{1}{2} \int_{0}^{x} S^{\prime \prime}(y) \log (x-y) d y+\frac{1}{2} \int_{x}^{L} S^{\prime \prime}(y) \log (y-x) d y \\
& -\frac{1}{2} \int_{0}^{x} S^{\prime}(y)\left\{e^{-\beta^{2}(x-y) \lambda^{-1}}-e^{-(x-y) \lambda^{-1}}\right\} \frac{d y}{(x-y)}
\end{aligned}
\end{aligned}
$$

The first two integral terms here are precisely the same as those which occur in the inert gas, subsonic flow, case, (Sears and Adams, 1953). The third integral, together with the logarithm term, summarises the effects of relaxation. Since an integral of this type is found to arise in other cases it is worthwhile giving it a special symbol. Thus we write

$$
I_{\beta}=-\frac{1}{2} \int_{0}^{x} S^{\prime}(y)\left\{e^{-\beta^{2}(x-y) \lambda^{-1}}-e^{-(x-y) \lambda^{-1}}\right\} \frac{d y}{(x-y)} .
$$


When the integral contains $\bar{\beta}$ or $B$ in place of $\beta$ we will denote this fact by an appropriate suffix, as in equation 50, In general we shall write it as $\mathrm{I}_{\alpha}$, where $\alpha$ may be $\beta, \bar{\beta}$ or $\mathrm{B}$.

A useful alternative form of $I_{\beta}$ is found by noting the definition of the exponential integral, $\mathrm{Ei}(-\mathrm{a})$. That is,

$$
E i(-a)=-\int_{a}^{\infty} e^{-\sigma} \sigma^{-1} d \sigma \text {. }
$$

Integrating equation 50 by parts with the upper limit equal to $x-\epsilon$ and then taking the limit as $\epsilon \rightarrow 0$ leads to,

$$
I_{\beta}=S^{\prime}(x) \log \beta+\frac{1}{2} \int_{0}^{x} S^{\prime \prime}(y)\left\{\operatorname{Ei}\left(\frac{y-x}{\lambda}\right)-\operatorname{Ei}\left(\beta^{2} \cdot \frac{y-x}{\lambda}\right)\right\} d y .
$$

We shall also fird some approximate forms of $I_{\beta}$ useful in later sections. Thus, when both $x / \lambda$ and $\beta^{2} x / \lambda \rightarrow 0$ we find that

$$
I_{\beta} \simeq \quad-\frac{\left(1-\beta^{2}\right)}{2 \lambda} \mathrm{S}(\mathrm{x}) \text {. }
$$

When both $x / \lambda$ and $\beta^{2} x / \lambda \rightarrow \infty$, it can be shown that ${ }^{*}$

$$
I_{\beta} \simeq S^{\prime}(x) \log \beta+\frac{\lambda}{2}\left(\beta^{-2}-1\right) S^{\prime \prime}(x) \text {. }
$$

Equation 53 is a suitable approximation for near-frozen flow (as $\lambda \rightarrow \infty$ ) and equation 54 is useful in near-equilibrium flow (as $\lambda \rightarrow 0$ ) provided that $\beta^{2}$ does not become so small that $\beta^{2} x / \lambda$ is no longer large. Thus equation 54 cannot be used too near to the beginning of the transonic regime. In the event that $x / \lambda \gg 1$ whilst $\beta^{2} x / \lambda<<1$, it can be shown that $\mathrm{I}_{\beta}$ behaves as follows,

$$
I_{\beta} \simeq-\frac{1}{2} \int_{0}^{x} S^{\prime \prime}(y) \log (x-y) d y+\frac{\beta^{2}}{2 \lambda} S(x)+\frac{1}{2} S^{\prime}(x) \log \lambda-\frac{1}{2} \operatorname{CS}^{\prime}(x)-\frac{\dot{\lambda}}{2} S^{\prime \prime}(x) .
$$

* Note that equation 54 always fails in some region sufficiently close to the nose of the body (where $x / L<1$ ) for any finite value of $\lambda$ however small. In such regions equation 53 is applicable. 
This result demonstrates how the solutions begin to break down as one approaches the "transonic" condition $\mathbb{M}_{e} \rightarrow 1$ (identical with $\beta \rightarrow 0$ ) in the limiting case of $\lambda \rightarrow 0$. In other words, if $\lambda=0$ and complete equilibrium prevails, the potential contains a logarithmic singularity in the limit $M_{e}=1$. However, when $\lambda \neq 0$ the linear solution continues smocthly up to the condition $\mathbb{M}_{e}=1$. We shall shortly show that it also passes smoothly and continuously through this condition and into the transonic regime.

Case (ii)

$$
\begin{aligned}
& 2 \pi \frac{b_{0}}{U}=S^{\prime}(x) \log \left(\beta_{f^{\prime}}(2)-\int_{0}^{x} S^{\prime \prime}(y) \log (x-y) d y\right. \\
& -\frac{1}{2} \int_{x}^{L} S^{\prime}(y) e^{-\beta^{2}(y-x) \lambda^{-1}} \frac{d y}{(y-x)}+\frac{1}{2} \int_{0}^{x} S^{\prime}(y) e^{-(x-y) \lambda^{-1}} \frac{d y}{(x-y)} .
\end{aligned}
$$

This result should be compared with the subsonic value of $\mathrm{b}_{0}$ given in equation 49. Using the notation defined in equation 50 et seq, equation 56 can be rearranged somewhat to read

$$
\begin{aligned}
& 2 \pi \frac{\mathrm{b}}{\mathrm{U}}=\mathrm{S}^{\prime}(\mathrm{x}) \log \left(\beta_{\mathrm{f}} / 2\right)-\int_{0}^{\mathrm{x}} \mathrm{S}^{\prime \prime}(\mathrm{y}) \log (\mathrm{x}-\mathrm{y}) \mathrm{dy}+\frac{1}{2} \int_{0}^{\mathrm{x}} \mathrm{S}^{\prime \prime}(\mathrm{y}) \log (\mathrm{x}-\mathrm{y}) \mathrm{e}^{-\bar{\beta}^{2}(\mathrm{x}-\mathrm{y}) \lambda^{-1}} \mathrm{dy} \\
& +\frac{1}{2} \int_{x}^{L} S^{\prime \prime}(y) \log (y-x) e^{-\bar{\beta}^{2}(y-x) \lambda^{-1}} d y \\
& -\frac{1}{2} \frac{\bar{\beta}^{2}}{\lambda} \int_{0}^{L} S^{\prime}(y) \log |x-y| e^{-\bar{\beta}^{2}|x-y| \lambda^{-1}} d y+\frac{I}{\beta}
\end{aligned}
$$

In this form it can fairly readily be seen that equations 49 and 57 become identical in the limits $\beta$ and $\bar{\beta}=0$. Thus $b_{0}$ passes smoothly into the transonic regime, provided that $0<\lambda<\infty$. It should be noted that equation 57 only follows from equation 56 if $\mathrm{S}^{\prime}(\mathrm{L})=0$. We assume $S^{\prime}(L)=0$ throughout Cases (i) and (ii). 
When the conditions $x / \lambda$ and $\overrightarrow{\beta^{2}} x / \lambda \ll 1$ are both satisfied, (note that this requires $\mathbb{M}_{f}$ not too near to 1 ), a suitable approximate form of $b_{0}$ is,

$$
\begin{gathered}
2 \pi \frac{b_{O}}{U} \simeq S^{\prime}(x) \log \left(\beta_{f} / 2\right)-\frac{1}{2} \int_{0}^{x} S^{\prime \prime}(y) \log (x-y) d y+\frac{1}{2} \int_{x}^{L} S^{\prime \prime}(y) \log (y-x) d x \\
-\left(\frac{1+\overline{\beta^{2}}}{2 \lambda}\right) S(x) .
\end{gathered}
$$

When the upper limit of the transonic range is approached, so that $\bar{\beta} \rightarrow \infty$, we may find a suitable approximate representation of $b_{0}$ under the conditions $\mathrm{x} / \lambda \ll 1, \overline{\beta^{2}} \mathrm{x} / \lambda \gg 1$, namely

$$
\begin{aligned}
& 2 \pi \frac{b_{0}}{U} \simeq S^{\prime}(x) \log \left(B_{e}(2)-\frac{1}{2} \int_{0}^{x} S^{\prime \prime}(y) \log (x-y) d y\right. \\
& \quad+\frac{1}{2} S^{\prime}(x)[C-\log \lambda]-\frac{1}{2} \frac{\lambda}{\beta^{2}} S^{\prime \prime}(x)-\frac{1}{2 \lambda} S(x) .
\end{aligned}
$$

Provided that we are not now too near to the lower end of the transonic range $\left(\mathbb{N}_{e}+1\right)$, we may be able to satisfy the conditions $x / \lambda$ and $\overline{\beta^{2}} \times / \lambda$ both $>1$. In that case, a suitable approximation for $b_{0}$ is $2 \pi \frac{b_{0}}{U} \simeq S^{\prime}(x) \log \left(B_{e} / 2\right)-\int_{0}^{x} S^{\prime \prime}(y) \log (x-y) d y-\frac{\lambda}{2}\left(1+\bar{\beta}^{-2}\right) S^{\prime \prime}(x)$

Finally we write down the "near-equilibrium" result at the lower end of the transonic range, i.e. $x / \lambda \gg 1, \bar{\beta}^{2} x / \lambda \ll 1$. This is

$$
\begin{gathered}
2 \pi \frac{\mathrm{b}}{\mathrm{U}} \cong \mathrm{S}^{\prime}(\mathrm{x}) \log \left(\beta_{\mathrm{f}} / 2\right)-\int_{0}^{\mathrm{x}} \mathrm{S}^{\prime \prime}(\mathrm{y}) \log (\mathrm{x}-\mathrm{y}) \operatorname{dy}+\frac{1}{2} \int_{\mathrm{x}}^{L} \mathrm{~S}^{\prime \prime}(\mathrm{y}) \log (\mathrm{y}-\mathrm{x}) \mathrm{dx} \\
-\frac{1}{2} \mathrm{~S}^{\prime}(\mathrm{x})[\mathrm{C}-\log \lambda]-\frac{\bar{\beta}^{2}}{2 \lambda} \mathrm{S}(\mathrm{x})-\frac{\lambda}{2} \mathrm{~S}^{\prime \prime}(\mathrm{x}) .
\end{gathered}
$$

Further comment on the behvaiou of $b_{0}$ in the transonic range will be postponed until we come to consider questions of drag in a later section. 


\section{Case (iii)}

In the supersonic regime it is found that

$$
2 \pi \frac{b_{0}}{U}=S^{\prime}(x) \log \left(B_{f} / 2\right)-\int_{0}^{x} S^{\prime \prime}(y) \log (x-y) d y+I_{B} \text {, }
$$

(see equation 50 et seq for the definition of $I_{B}$ ).

is identical with $I_{\beta}$, except that $B$ is now written for $\beta$, it follows that we can write

$$
I_{B} \simeq \frac{B^{2}-1}{2 \lambda} \cdot S(x)
$$

when $x / \lambda$ and $B^{2} x / \lambda$ both $\rightarrow 0$ and

$$
I_{B} \approx S^{\prime}(x) \log B-\frac{\lambda}{2}\left(1-B^{-3}\right) S^{\prime \prime}(x),
$$

when both $x / \lambda$ and $B^{2} x / \lambda \rightarrow \infty$

The approximation in equation 63 is nor suitable if $\mathrm{B}^{2}$ becomes too large (i.e. we are too near to $M_{f}=1$ ). In the event that $x / \lambda \ll 1$ whilst $B^{2} x / \lambda>$ : we find that

$$
\begin{aligned}
2 \pi \frac{b_{o}}{U} \simeq & S^{\prime}(x) \log \left(B_{e} / 2\right)-\frac{1}{2} \int_{0}^{x} S^{\prime \prime}(y) \log (x-y) d y+\frac{1}{2} S^{\prime}(x)[C-\log \lambda] \\
& +\frac{\lambda}{2 B^{2}} S^{\prime \prime}(x)-\frac{1}{2 \lambda} S(x) .
\end{aligned}
$$

Comparing this result with equation 59 it can be seen that they become identical in the limit $\bar{\beta} \rightarrow \infty, \mathrm{B} \rightarrow \infty$. It can be shown that the values of $b_{o}$ in the transonic and supersonic cases coincide for all $\lambda$ when $\mathrm{MI}_{\mathrm{f}}=1$, so that a smooth transition from transonic to supersonic states occurs. (See also equations 60 and 62 with 64 when $\bar{\beta}^{2} \rightarrow \infty$ and $\mathrm{B}^{2} \rightarrow \infty$ ). 


\section{Drag}

Having obtained values for $b_{0}$ it is now possible to evaluate the drag of bodies in a relaxing gas flow, making use of Ward's formula (written out in full, except for base drag, in equation 46). It has been remarked earlier that the sub-and transonic problems must concern themselves only with bodies pointed at both ends. This condition can be relaxed in the fully supersonic regime. We shall deal with the two classes of body shape separately.

\section{(a) Doubly-Pointed Bodies}

Fiere we can use the simpler formula in equation 48 . Writing

$$
\mathrm{D}=\frac{1}{2} \rho_{\infty} \mathrm{U}^{2} \mathrm{~S}_{\mathrm{r}} \mathrm{C}_{\mathrm{D}}
$$

where $\mathrm{S}_{\mathrm{r}}$ is some suitable reference area and $\mathrm{C}_{\mathrm{D}}$ is the drag coefficient, we proceed to find values of $C_{D}$ in each of the cases (i), (ii) and (iii) treated above.

Case (i)

Using equation 47 for $a_{0}$, it can readily be shown that the first two integrals in equation 49 combine to give a zero contribution to $\mathrm{C}_{\mathrm{D}}$ in all circumstances, as indeed does the first term in equation 49. Turning to the last integral in equation 49 , narnely $I_{\beta}$, we can obtain a compact gereral result via the form of $I_{\beta}$ given in equation 52 . The first term in equation 52 contributes nothing to the final value of $C_{D}$ and we are left with

$$
S_{r} C_{D}=\frac{1}{2 \pi} \int_{0}^{L} S^{\prime \prime}(x) \int_{0}^{x} S^{\prime \prime}(y)\left\{E i\left(\frac{y-x}{\lambda}\right)-E i\left(\beta^{2} \cdot \frac{y-x}{\lambda}\right)\right\} d y d x .
$$

or, alternatively,

$S_{r} C_{D}=\frac{1}{4 \pi} \int_{0}^{L} \int_{0}^{L} S^{\prime \prime}(x) S^{\prime \prime}(y)\left\{E i\left(-\frac{|x-y|}{\lambda}\right)-E i\left(-\beta^{2} \frac{|x-y|}{\lambda}\right)\right\} d y d x$. 
It is interesting to compare this result with the wave drag result for the supersonic flow past doubly-pointed bodies (with no relaxation effects) obtained by Ward and others. (We shall obtain this result in examining case (iii) below; see equation 77). Here the kernel function of the drag integral is made up of the exponential integral terms in place of the simple logarithm. Equation 67 contrasts with the non-relaxing subsonic flow problem, which would yield zero drag since it includes no dissipative mechanism to give rise to a drag. Clearly the $C_{D}$ predicted by equations 67 is always positive; for example, in the "near-frozen" case we may use the approximation in equation 53 to show that

$$
S_{r} C_{D} \simeq \frac{\left(1-\beta^{2}\right)}{2 \pi \lambda} \int_{0}^{L} S^{\prime}(x) d x,
$$

whilst in "near-equilibrium" flow ( $\beta$ not too near zero) equation 54 shows that ${ }^{*}$

$$
S_{r} C_{D} \approx \frac{\lambda\left(\beta^{-2}-1\right)}{2 \pi} \int_{0}^{L} \cdot S^{\prime \prime 2}(x) d x .
$$

The fact that $\beta$ is $<1$ in subsonic flow then guarantees that $C_{D}$ is positive.

Since $C_{D} \Rightarrow 0$ in either limiting case $\lambda \rightarrow 0$ or $\lambda \rightarrow \infty$, and is positive between these limits, it follows that there must be a maximum value of $C_{D}$ somewhere between $\lambda=0$ and $\infty$. The value of $\lambda$ which will make the "relaxation" drag a maximum will depend on both the body

* In using equation 54 to evaluate drag in the "near-equilibrium" case it is implied that the errors introduced into $S_{r} C_{D}$ by ignoring the failure of equation 54 near $x=0$ are negligible. This is a fairly restrictive condition, as can be appreciated from an examination of the exact value of $\mathrm{I}_{\beta}$ in the case of a parabolic arc body, treated in this section below. Equation 69 and corresponding expressions derived in Cases (ii) and (iii) are sufficient for a discussion of generalities but must be used with care when deriving approximate numerical results. 
shape and the value of $\beta^{2}$. It does not seem possible to find this value analytically, either in general terms or for a particular body shape, but one would suspect that it should be such as to make $(L / \lambda)$ roughly of order one.*

In the event that $\beta^{2}+0$ whilst $\lambda$ is small, the approximation in equation 55 becomes valid, giving

$$
S_{r} C_{D} \approx-\frac{1}{2 \pi} \int_{0}^{L} S^{\prime \prime}(x) \int_{0}^{x} S^{\prime \prime}(y) \log (x-y) d y d x-\frac{\beta^{2}}{2 \pi \lambda} \int_{0}^{L} S^{\prime 2}(x) d x
$$

The appearance of the double integral term in this expression is interesting, since it is precisely one half of the supersonic wave drag for this class of body shape. In the equilibrium flow limit at $\mathrm{NI}_{e}=1\left(\beta^{2}=0\right)$, the present theory therefore puts the "transonic" drag at just this value. Practically of course, the result is incorrect since the original partial differential equation (equation 27) fails to describe the flow field properly for $\mathbb{M}_{\mathrm{e}}=1$ and $\lambda=0$. When $\lambda$ is not zero we may expect that equation 70 has some validity however, because in this condition there is a mechanism present for preventing the "piling -up" of acoustic disturbances which leads to the breakdown of linear solutions when $M_{e}=1$ and $\lambda=0$. A similar situation is encountered in the case $\mathbb{M}_{f}=1, \lambda=\infty$.

Case (ii)

In the transonic case it is best to use the result in equation 56 to find the drag. After some rearrangement, it can be shown that $S_{r} C_{D}=-\frac{1}{\pi} \int_{0}^{L} S^{\prime \prime}(x) \int_{0}^{x} S^{\prime \prime}(y) \log (x-y) d y d x+\frac{1}{\pi} \int_{0}^{L} S^{\prime \prime}(x) \int_{0}^{x} S^{\prime \prime}(y)$. $\operatorname{Ei}\left(\bar{\beta}^{2} \frac{(y-x)}{\lambda}\right) d y d x+\frac{1}{2 \pi} \int_{0}^{L} S^{\prime \prime}(x) \int_{0}^{x} S^{\prime \prime}(y)\left\{\operatorname{Ei}\left(\frac{y-x}{\lambda}\right)-\operatorname{Ei}\left(\bar{\beta}^{2} \frac{(y-x)}{\lambda}\right)\right\} d y d x$. (71)

But see numerical example later in this section. 
Comparing this with the result for the subsonic case (equation 67 ) it can be seen that a term exactly equivalent to $S_{r} C_{D}$ (subsonic) occurs, the oniy difference being the appearance of $\bar{\beta}$ for $\beta$. Now, however, the drag is reinforced by the presence of a full supersonic wave drag contribution (first integral in equation 71), combined with a further relaxation term. To distinguish between the various contributions to $C_{D}$ in the present case, we shall refer to the first two integral terms in equation 71 as the "transonic wave drag" and to the last integral as the "relaxation drag". This division is simply for convenience later: note that the "transonic wave drag" in fact includes relaxation terms. We recall that $0 \leqslant \bar{\beta}^{2} \leqslant \infty$.

When $\lambda \rightarrow \infty$ and we are not too near to $\bar{\beta}=\infty$ it is found that the transonic wave drag contributes a term

$$
\frac{\overline{\beta^{2}}}{\pi \lambda} \int_{0}^{L} s^{\prime 2}(x) d x
$$

to $S_{r} C_{D}$, whilst the relaxation drag part behaves like

$$
\frac{1-\bar{\beta}^{2}}{2 \pi \lambda} \int_{0}^{L} S^{\prime 2}(x) d x \text {. }
$$

The net effect is to give rise to a positive value of $C_{D}$ of course, but it is interesting to note that the relaxation drag contribution falls to zerc and then becomes negative as $\bar{\beta}$ passes through the value unity. That expression $72 \mathrm{~b}$ is part of a general result valid for all values of $\lambda$ can readily be seen from equation 71 . The changeover point occurs when

$$
U \simeq\left(a_{100} a_{200}\right)^{\frac{1}{2}}
$$

roughly speaking, if $a_{100}$ and $a_{200}$ do not differ greatly. Meanwhile, the transonic wave drag continues to increase as $\bar{\beta}$ increases, as indeed does the net drag. 
Still retaining the assumption $\lambda \rightarrow \infty$, but now imagining that $\bar{\beta}^{2}$ has become so large that $\bar{\beta}^{2} L / \lambda \rightarrow \infty$, we find that the two parts of $S_{r} C_{D}$ behave like

$$
-\frac{1}{\pi} \int_{0}^{L} S^{\prime \prime}(x) \int_{0}^{x} S^{\prime \prime}(y) \log (x-y) d y d x-\frac{\lambda}{\pi \overline{\beta^{2}}} \int_{0}^{L} S^{\prime \prime 2}(x) d x
$$

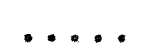

$+\frac{\lambda}{2 \pi \bar{\beta}^{2}} \int_{0}^{L} S^{\prime \prime 2}(x) d x+\frac{1}{2 \pi} \int_{0}^{L} S^{\prime \prime}(x) \int_{0}^{x} S^{\prime \prime}(y) \log (x-y) d y d x+\frac{1}{2 \pi \lambda} \int_{0}^{L} S^{\prime 2}(x) d x$ respectively. The transonic wave drag in equation 74 a continues to increase as $\bar{\beta}$ increases to its final value in the present conditions and, in line with the comments made above about the relaxation drag, this part $(74 b)$ is found to be negative. The double integral term in equation $74 a$ is the full supersonic wave drag for a doubly-pointed body in a nonrelaxing gas flow (essentially a positive contribution to $\mathrm{S}_{r} \mathrm{C}_{D}$ ). It seems clear from the results $72 \mathrm{a}$ and $74 \mathrm{a}$ that the transonic wave drag increases steadily from zero at the subsonic $\left(M_{e}=1\right)$ end of the transonic range up to the full supersonic value at its upper end $\left(\mathbb{M}_{f}=1\right)$, a result which we will confirm below in the other extreme of $\lambda \rightarrow 0$.

We observe also (at least when $\lambda+\infty$ ) that the net value of $S_{r} C_{D}$ on passing out of the transonic regime $(\bar{\beta}=\infty)$ is a little greater than one half of the full supersonic wave drag without relaxation effects. However the Iinear solution breaks down at $M_{f}=1$ when $\lambda \rightarrow \infty$ on account of the logarithmic singularity arising in $b_{0}$ (see equation 59 ).

When $\lambda \rightarrow 0$ suitable approximate forms of the transonic wave and relaxation drags are, in the former case exactly equation $74 \mathrm{a}$ and in the latter case

$$
\frac{\lambda}{2 \pi}\left(\bar{\beta}^{-2}-1\right) \int_{0}^{L} S^{\prime \prime 2}(x) d x
$$


provided $\bar{\beta}$ is not too near to zero. In the event that $\overline{\beta^{2}} L / \lambda \rightarrow 0$ despite $L / \lambda$ being $>1$, we find that the two parts of $C_{D}$ are given by equation $72 a$ for the transonic wave drag and

$-\frac{1}{2 \pi} \int_{0}^{L} S^{\prime \prime}(x) \int_{0}^{x} S^{\prime \prime}(y) \log (x-y) d y d x-\frac{\bar{\beta}^{2}}{2 \pi \lambda} \int_{0}^{L} S^{\prime 2}(x) d x-\frac{\lambda}{2 \pi} \int_{0}^{L} S^{\prime \prime 2}(x) d x$

.....

for the relaxation drag. Note that the sum of expressions $72 \mathrm{a}$ and 76 gives a value of $\mathrm{S}_{\mathrm{r}} \mathrm{C}_{\mathrm{D}}$ exactly equal to that in equation 70 when both $\beta^{2}$ and $\bar{\beta}^{2}$ are zero. When $\beta^{2}=\vec{\beta}^{2} \neq 0$, however, the net transonic drag is greater than the subsonic value, as we should expect. That a similar state of affairs arises when $\lambda$ is large follows from comparison of equation 68 with the sum of expressions $72 a$ and $72 b$.

An explanation of the negative contribution to the overall drag which is provided by the relaxation term for $\bar{\beta}^{2}>1$ will be deferred until the Section on pressure distributions which is to follow, but we remark here that it appears to be a characteristic of the pointed tail body shape required in the sub - and transonic regimes by the present theory.

\section{Case (iii)}

Entering the fully supersonic regime the relevant value of $b_{0}$ is found in equation 62 and it follows the+

$$
\begin{aligned}
S_{r} C_{D} & =-\frac{1}{\pi} \int_{0}^{L} S^{\prime \prime}(x) \int_{0}^{x} S^{\prime \prime}(y) \log (x-y) d y d x \\
& -\frac{1}{2 \pi} \int_{0}^{L} S^{\prime \prime}(x) \int_{0}^{x} S^{\prime \prime}(y)\left\{\operatorname{Ei}\left(B^{2(y-x)} \frac{(y)}{\lambda}\right)-E i\left(\frac{y-x}{\lambda}\right)\right\} d y d x .
\end{aligned}
$$

$\mathrm{B}^{2}$, in the "relaxation" drag integral in equation 77 , is $>1$. The contribution made by this integral to the net $C_{D}$ value is negative, as we shall confirm below. The first integral in equation 77 is the full supersonic 
wave drag for a non-relaxing gas flow.

When $\lambda \rightarrow \infty$ and we are not too near to $\mathbb{M}_{f}=1$, the relaxation drag is approximately

$$
-\frac{\left(B^{2}-1\right)}{2 \pi \lambda} \int_{0}^{L} S^{\prime 2}(x) d x
$$

whilst, when $\lambda \rightarrow 0$, it contributes a term

$$
-\lambda \frac{\left(1-B^{-2}\right)}{2 \pi} \int_{0}^{L} S^{\prime \prime 2}(x) d x
$$

for all values of $\mathrm{B}^{2}$.

If $\lambda \rightarrow \infty$, but $B^{2}$ becomes so large despite this that $B^{2} L / \lambda>1$, we find that the relaxation drag behaves like

$$
\frac{1}{2 \pi} \int_{0}^{L} S^{\prime \prime}(x) \int_{0}^{x} S^{\prime \prime}(y) \log (x-y) d y d x+\frac{\lambda}{2 \pi B^{2}} \int_{0}^{L} S^{\prime \prime 2}(x) d x+\frac{1}{2 \pi \lambda} \int_{0}^{L} S^{\prime 2}(x) d x .
$$

When $B^{2}=\infty$, equation 80 plus the full supersonic wave drag gives a result for $\mathrm{S}_{\mathrm{r}} \mathrm{C}_{\mathrm{D}}$ which agrees with the sum of expressions $74 \mathrm{a}$ and $74 \mathrm{~b}$ for $\bar{\beta}^{2}=\infty$. The same agreement occurs in the near-equilibrium state, for putting $\bar{\beta}^{2}=\infty$ in the sum of equations 74 a and 75 gives exactly the result found by adding equation 79 with $B^{2}=\infty$ to the full supersonic wave drag.

In the supersonic regime, the effect of relaxation is such as to give $C_{D}$ values which are always less than the non-relaxing wave drag on doubly-pointed bodies.

To conclude this section on doubly-pointed bodies we consider a specific example, namely the body whose meridian profile is a parabolic arc. We only examine the sub- and supersonic regimes since, for most practical cases, the transonic regime is very narrow and the solutions that 
we have obtained in this region are of rather more academic than practical interest. We shall write

$$
S_{r} C_{D r}=\frac{1}{2 \pi} \int_{0}^{L} S^{\prime \prime}(x) \int_{0}^{x} S^{\prime \prime}(y)\left[E i \frac{(y-x)}{\lambda}-E i\left(\alpha^{2} \frac{y-x}{\lambda}\right)\right] d y d x
$$

where $\alpha^{2}$ may be either $\beta^{2}$ or $B^{2}$, as the case may be. $C_{D r}$ will be referred to as the relaxation drag coefficient. The method of evaluation of the integral in equation 81 is outlined in Appendix B. Equation B13 gives the equation for the meridian profile and equation B15 gives the value of $\mathrm{C}_{\mathrm{Dr}}$ for this body shape, assuming that the reference area $\mathrm{S}_{\mathrm{r}}$ is equal to the maximum body cross-sectional area. Note that in deriving equation $B 15$ the body length $L$ has been set equal to one (no loss of generality is incurred by this), so that $\lambda$ there is $\tau U$ measured in units of body length.

Fig. 1 shows $2(32 \delta)^{-2}\left|C_{D r}\right|$ plotted against $\lambda$ for two values of $\alpha^{2}$, namely 0.574 and 1.327. These values correspond to $\mathrm{M}_{f}$ 's of 0.9 and 1.2 respectively, with the square of the sound speeds ratio equal to 1.1 . This latter quantity is roughly that which occurs in $\mathrm{CO}_{2}$ at temperatures somewhat greater than $288^{\circ} \mathrm{K}$. For this linear triatomic molecule the relaxing internal mode is that involving transverse vibrations and at N.T.P. the relaxation time is about $10 \mu \mathrm{sec}$. We note that the maxima for $\left|C_{D r}\right|$ occur for $\lambda^{\prime}$ 's between 0.1 and 0.3 body lengths, so that with values of $U$ of about $3 \times 10^{4} \mathrm{~cm}$. $/ \mathrm{sec}$. we find that the body lengths to give this maximum are roughly of order $\left(0.3 / \mathrm{p}_{\mathrm{a}}\right) \mathrm{cm} \cdot\left(\mathrm{p}_{\mathrm{a}}\right.$ is free stream pressure measured in atmospheres). In this particular case then, even pressures as high as $10^{-1}$ atm. can lead to maxima in $\left|C_{\mathrm{Dr}}\right|$ for body sizes comparable to those found in a number of experimental facilities. It is not possible to state in general terms just how significant $\mathrm{C}_{\mathrm{Dr}}$ is in relation to skin friction and form drag, since thest are viscous effects and 
hence outside the scope of the present theory. However, under conditions like those just described for the occurrence of $\left|\mathrm{C}_{\mathrm{Dr}}\right|_{\max }$, the Reynolds number based on body length should be of order $10^{6}$. Thus the laminar friction drag coefficient based on body surface area will be of order $10^{-3}$ which, for a slender body, is roughly a $C_{D}$ of $10^{-3} \delta^{-1}$ when the reference area is $\pi \delta^{2}$ (as in the definition of $\mathrm{C}_{\mathrm{Dr}}$ ). Allowing for the crudeness of the estimated friction drag coefficient it is seen that $\left|\mathrm{C}_{\mathrm{Dr}}\right|_{\max }$ may be comparable with it in some circumstances. For a well streamlined body, on which friction drag predominates, it seems safe to conjecture that relaxation drag may cause significant increases in overall drag in subsonic flow. Clearly each special case must be examined in detail, but the foregoing estimates do tend to suggest that such examination is worthwhile.

In supersonic flow, of course, the drag picture is dominated by the appearance of wave drag. For the case of the parabolic arc body the wave drag coefficient $\left(C_{D W}\right)$ is found to be given by

$$
2(32 \delta)^{-2} C_{D W}=\frac{1}{12} \text {, }
$$

and clearly this is much larger than the values of $\left|C_{D r}\right|$ shown in Fis. 1. We note that $\left|C_{\mathrm{Dr}}\right|$ may still be comparable with friction drag
coefficients.

In conclusion we note that the amplitude of the effects of relaxation will increase as $\alpha^{2}$ increases. Broadly speaking this implies that the largest effects will arise in the transonic regime, but, for obvious reasons, the present linear theory cannot be used to obtain numerical results of convincing accuracy in that Mach number range. $a^{2}$ is increased at any $\mathbb{M}_{f}$ value by increasing the speeds of sound ratio $\left(a_{1} / a_{2}\right)$. 
A maximum value of $\left(a_{1} / a_{2}\right)^{2}$ (namely $\left.25 / 21\right)$ occurs for rotational relaxation in a diatomic gas, but we note that much larger values can arise when chemical reactions are involved (e.g. Clarke, 1958).

(b) Blunt Based Bodies

Confining ourselves to the fully supersonic regime in order to be able to deal with this particular class of body shapes, equations 46,47 and 62 combine to show that

$$
\begin{aligned}
S_{\mathrm{r}} C_{D}= & -\frac{1}{\pi} \int_{0}^{L} S^{\prime \prime}(x) \int_{0}^{x} S^{\prime \prime}(y) \log (x-y) d y d x+\frac{S^{\prime}(L)}{2 \pi} \int_{0}^{L} S^{\prime \prime}(y) \log (L-y) d y \\
& +\frac{1}{2 \pi} \int_{0}^{L} S^{\prime \prime}(x) \int_{0}^{x} S^{\prime \prime}(y)\left\{E i\left(\frac{y-x}{\lambda}-\right)-E i\left(B^{2} \cdot \frac{y-x}{\lambda}\right)\right\} d y d x \quad(82) \\
& -\frac{S^{\prime}(L)}{4 \pi} \int_{0}^{L} S^{\prime \prime}(y)\left\{E i\left(\frac{y-L}{\lambda}\right)-E i\left(B^{2} \cdot \frac{y-L}{\lambda}\right)\right\} d y-\frac{1}{U^{2}}\left(\int_{C} \frac{\partial \phi}{\partial v} d \sigma\right)
\end{aligned}
$$

$\left(C_{D}\right.$ is defined as in equation 66 above). The final integral here can only be evaluated once the particular body shape and attitude is given. For example, it is this integral which determines the effects of body incidence on $\mathrm{C}_{\mathrm{D}}$. Since the cross-flow problem is unaffected by relaxation to the present order of approximation we will have no need to consider questions of body attitude, for the results will be identical with those already obtained by Ward. Thus we can confine our attention to the "gero-incidence" behaviour of the integral in question, incidence being measured from the body position giving zero cross wind force.

In particular, we examine the body of revolution, for which the normal derivative $\partial \phi / \partial v$ becomes simply $\partial \phi / \partial r$ and the boundary condition gives

$$
\frac{\partial \phi}{\partial r}=U R^{\prime}(x)
$$


on the contour $C$. We write $R(x)$ for body radius, the prime denoting differentiation with respect to $x$, as usual. In these circumstances, the disturbance potential is

$$
\phi(x, r)=U \frac{S^{\prime}(x)}{2 \pi} \log r+b_{0}(x),
$$

as there is no dependence on the angle $\theta$ and it then follows that

$$
-\frac{1}{U^{2}}\left(\int_{C} \phi \frac{\partial \phi}{\partial v} d \sigma\right)_{x=L}=-\frac{S^{\prime 2}(L)}{2 \pi} \log R(L)-S^{\prime}(L) \frac{b_{0}(L)}{U}
$$

Taking the value of $b_{o}$ from equation 62 and using the form of $I_{B}$ given in equation 52 , the sum of wave plus relaxation drag for a bluntbased body of revolution at zero incidence is found to be

$$
\begin{aligned}
S_{r} C_{D}= & -\frac{S^{\prime 2}(L)}{2 \pi} \log \left(B e_{e} R(L) / 2\right)-\frac{1}{\pi} \int_{0}^{L} S^{\prime \prime}(x) \int_{o}^{x} S^{\prime \prime}(y) \log (x-y) d y d x \\
& +\frac{S^{\prime}(L)}{\pi} \int_{0}^{L} S^{\prime \prime}(y) \log (L-y) d y \\
+ & \frac{1}{2 \pi} \int_{0}^{L} S^{\prime \prime}(x) \int_{0}^{x} S^{\prime \prime}(y)\left\{E i\left(\frac{y-x}{\lambda}\right)-E i\left(B^{2} \cdot \frac{y-x}{\lambda}\right)\right\} d y d x \\
- & \frac{S^{\prime}(L)}{2 \pi} \int_{0}^{L} S^{\prime \prime}(y)\left\{E i\left(\frac{y-L}{\lambda}\right)-E i\left(B^{2} \cdot \frac{y-L}{\lambda}\right)\right\} d y .
\end{aligned}
$$

Notice the appearance of the factor $\mathrm{B}_{\mathrm{e}}$ in the first term of equation 86 . Because this term arises naturally in the expression for $C_{D}$ we shall take the equilibrium state as the basis for comparison in what follows. The first three terms in equation 86 will be referred to as the drag occurring in a non-relaxing gas; the last two integrals therefore summarise the relaxation effects.

In the near equilibrium state, these relaxation terms behave like

$$
-\lambda \frac{\left(1-B^{-2}\right)}{2 \pi}\left\{\int_{0}^{L} S^{\prime \prime 2}(x) d x-S^{\prime}(L) S^{\prime \prime}(L),\right.
$$


to a first order in $\lambda$. When $\lambda \rightarrow \infty$ (and $B^{2}$ is not too large) they are approximately

$$
\frac{S^{\prime 2}(L)}{2 \pi} \log B-\frac{B^{2}-1}{2 \pi \lambda} \int_{0}^{L} S^{12}(x) d x .
$$

This last result confirms that, in the limit $\lambda=\infty$, the value of $S_{r} C_{D}$ becomes the one appropriate to a gas flow at the frozen Mach number $\mathrm{Mi}_{f}$, since the logarithm here combines with that in the non-relaxing gas flow to give a term $-S^{\prime 2}(L)(2 \pi)^{-1} \log \left(B_{f} R(L) / 2\right)$. This result also indicates that $\mathrm{S}_{\mathrm{r}} \mathrm{C}_{\mathrm{D}}$ is always somewhat less than the "frozen Mach number" value.

It is not so easy to deduce what is happening to $S_{r} C_{D}$ in the nearequilibrium state, since its behaviour will depend on the relative magnitude of the terms $S^{\prime}(L) S^{\prime \prime}(L)$ and the integral in equation 87 . We may expect that the behaviour depends on body shape and it seems reasonable to use the parabolic arc body of the previous numerical example to indicate roughly what this may be. If this body shape is assumed to terminate at $\mathrm{x}=3 \mathrm{~L} / 4 \mathrm{we}$ find that the curley bracket term in equation 87 has the value $+\left(32 \pi \delta^{2}\right)^{2} \mathrm{~L},(81 / 640)$; when the body ends at $x=L / 2$ it has the value $+\left(32 \pi \delta^{2}\right)^{2} L(1 / 10)$ and when the body ends at $L / 4$ it has the value $-\left(32 \pi \delta^{2}\right)^{2} \mathrm{~L}(3 / 32)$. In the last case, where $S^{\prime}(x)$ is definitely positive at the base, the drag is greater than the non-relaxing gas value, but somewhere between this condition and the one which gives $S^{\prime}(x)=0$ at the base the relaxation drag increment passes through zero and becomes negative. It would seem reasonable to suppose that it is always negative for bodies with boat-tailing.

To summarise briefly then, we always expect to find drag less than the frozen Mach number value. Depending on the body shape and the values of $\lambda$ and $B^{2}$ we may even find drag somewhat less than the equilibrium 
flow value. As $\lambda$ increases in such a case it seems reasonable to suppose that the net drag will eventually begin to increase, become equal to the equilibrium value once more (this time at a finite, non-zero value of $\lambda$ ) and thence lie between the equilibrium and frozen flow values of $C_{D}$ for all higher values of $\lambda$. It is worth noting the difference between the (quite complicated) drag behaviour deduced here and that found by Vincenti (1960) for the wavy wall. In the latter case $C_{D}$ always lies between the equilibrium and frozen Nach number values in the supersonic regime, probably on account of the multiplicity of reflected disturbances emanating from upstream regions of the flow.

The simplest example of a blunt-based body is the right-circular cone and we proceed to give some numerical results for this shape.

With a body length of unity, we take

$$
R(x)=\delta x
$$

for the cone. Whence it follows that $S^{\prime \prime}(x)=2 \pi \delta^{2}$. Some of the results in Appendix $B$ can be used to evaluate the integrals occurring in equation 86 and it is found that the relaxation drag coefficient $C_{D r}$ is given by

$$
\begin{aligned}
& \left.\delta^{-2} C_{D r}=\lambda^{2}\left(1-B^{-4}\right)-\lambda^{2} f e^{-1 / \lambda}-B^{-4} e^{-B^{2} / \lambda}\right) \\
& -\lambda\left(e^{-1 / \lambda}-B^{-2} e^{-B^{2} / \lambda}\right)+E_{1}(1 / \lambda)-E_{1}\left(B^{2} / \lambda\right) .
\end{aligned}
$$

(The notation is explained in Appendix B). The wave drag contribution to the total $C_{D}$ is giren by

$$
\delta^{-2} C_{D W}=2 \log \left(2 / B_{e} \delta\right)-1 \text {. }
$$

where we write $C_{D w}$ for the wave drag ooefficient. All drag coefficients have used a value of $S_{r}=\pi \delta^{2}$. 
Fig. 2 shows $\delta^{-2} C_{D r}$ plotted against $\lambda$ in units of body length for the value $B^{2}=1.223$. With the square of the speeds of sound ratio equal to 1.1 this corresponds to an $M_{e}$ of $\sqrt{2}$.

The presence of the factor $\log \left(2 / \mathrm{B}_{\mathrm{e}} \delta\right)$ in $\mathrm{C}_{\mathrm{DW}}$ makes the ratio of $\mathrm{C}_{\mathrm{Dr}}$ to wave drag dependent on the thickness ratio of the body. For example, when $\delta=\tan 5^{\circ}, \delta^{-2} C_{D W}=5.24$. We see that, as in the case of the parabolic arc body at supersonic speeds, the contribution made by relaxation effects is very small in comparison with the wave drag.

The cone is a further example of the type of body for which $\mathrm{C}_{\mathrm{Dr}}$ is always of one sign for any value of $\lambda$ (compare the "one-quarter" parabolic arc body mentioned above).

To conclude this section on drag, we may summarise the results briefly by saying that the effects of relaxation depend quite strongly on actual body shape. It seems likely that such effects are comparable with skin friction drag but do not seem to approach the magnitude of wave drag coefficients.

\section{The Pressure Coefficient}

The pressure coefficient is defined in equation 29 , which also shows the approximate relation between C.P. and the disturbance potential in the present, linear, theory. We are rether more interested in the differences which arise as a result of relaxation effects and it is clear from equations 29 and 44 that this only involves the difference between the relevant $b_{0}(x)$ functions. In fact, writing (C.P.) ${ }_{r}$ and (C.P.) for the pressure coefficients with and without relaxation effects, respectively, with a similar suffix notation for the $b_{0}$ functions, it is found that

$$
\Delta(C \cdot P)=(C \cdot P)_{r}-(C \cdot P)_{e}=-\frac{2}{U}\left(b_{o r}^{\prime}-b_{o e}^{\prime}\right) \text {. }
$$


(The prime on $b_{0}$ indicates differentiation with respect to $x$ ). To the present approximation, equation 92 is true for all shapes and attitudes which come within the classification "slender body". (C.P.) $\mathrm{e}$ chosen to be the pressure coefficient under full equilibrium conditions (i.e. the singular case, $\lambda=0$ ).

We shall not consider the transonic regime here and, this being so, it follows that

$$
\Delta \text { (C.P.) }=-\frac{1}{\pi}\left(I_{\alpha}^{\prime}-S^{\prime \prime}(x) \log \alpha\right),
$$

where $\alpha$ can be either $\beta$ or $B$, as the case may be. (Equation 93 follows from the results for $\mathrm{b}_{\mathrm{o}}$ given in equation 49 and 62 , together with the definition of $I_{\alpha}$ in equations 50 and 52 ). Some of the approximate forms of $I_{\alpha}$ derived in Section 4 can now be used to indicate roughly how $\Delta$ (C. P.) behaves in different situations. (One can verify that the derivative of the approximate form of $I_{\alpha}$ is the same as the approximate form of the derivative). Thus, when $x / \lambda \rightarrow 0$ equations 53 and 63 show that

$$
\pi \cdot \Delta(\text { C. P. }) \simeq \frac{1-\alpha^{2}}{2 \lambda} \cdot S^{\prime}(x)+S^{\prime \prime}(x) \log \alpha
$$

and when $x / \lambda \rightarrow \infty$, equations 54 and 64 show that

$$
\pi \Delta\left(\mathrm{C} . \mathrm{P}_{.}\right) \simeq \frac{\lambda}{2}\left(1-\alpha^{-2}\right) \mathrm{S}^{\prime \prime \prime}(\mathrm{x}) \text {. }
$$

Since equation 94 will be a valid approximation for sufficiently small $x$ for any value of $\lambda$, other than the singuiar value $\lambda=0$, we infer that C.P. at the nose of the body always equals the frozen flow value. The only exception occurs in the singular case $\lambda=0$, when of course $\Delta\left(C . P_{.}\right)=0$ everywhere, as can be seen from equation 95 . In general then, a relaxation zone, starting from the frozen C.P. value begins to form at the nose of the body and this is true whether the flow be subsonic or supersonic. Equation 95 indicates that the cone, for which 
$S^{\prime \prime \prime}(x)=0$, is rather a special case insofar as no relaxation effects will occur in the near equilibrium state $(\lambda \rightarrow 0)$ in regions where $x / \lambda \gg 1$.

It is interesting to note that the present theory indicates no "upstream influence" of relaxation effects on C.P. in subsonic flow. Indeed the integral term $I_{\beta}$ (and hence its derivative with respect to $x$ ), vanishes for all $x<0$. Since $S^{\prime \prime}(x)$ is also zero for $x<0$ it follows from equation 93 above that C.P. has its equilibrium value upstream of $x=0$. That upstream influence on C.P. does exist in subsonic flow follows from the behaviour of the second integral in equation 49 . When $x<0$ this integral can be written as

$$
\frac{1}{2} \int_{0}^{L} S^{\prime \prime}(y) \log (y+|x|) d y \neq 0 .
$$

$\circ$

It will shortly be shown that similar conclusions do not follow about the relaxation effects on the translational and internal mode temperatures in subsonic flow. It would seem reasonable to conclude that the lack of upstream relaxation eiffect upon C.P. in subsonic flow is a characteristic of the linear theory and that it would therefore be safer to say, such effect as might exist for C.P. is at most of second order. In supersonic flow there is no such difficulty since upstream influence (quite properly) does not exist at all.

Returning to the approximate $\Delta$ (C.P.) values in equations 94 and 95 , we observe first the change in sign of the terms involving $\alpha$ in the two Mach number regimes. These changes of sign are consistent with the relaxation drag behaviour in subsonic and supersonic flow and indicate in fact how this comes about. For subsonic flow with $L / \lambda \rightarrow 0$ (remembering that $\alpha=\beta<1$ ) equation 94 shows that it is the term in $S^{\prime}(x)$ which produces the relaxation drag, since that in $S^{\prime \prime}(x)$ has zero net effect on the drag of 
the doubly pointed bodies required in the subsonic flow case, In supersonic flow under the condition $L / \lambda \rightarrow 0$ the influence of this $S^{\prime \prime}(x)$ term begins to be felt on the blunt based bodies permitted in this regime.

The influence of body shape on the pressure shifts arising from relaxation effects is clear from equations 94 and 95 . It is also clear that each particular case must be treated on its merits. What is not quite so clear is how these effects are brought about. The most plausible explanation seems to be just the one advanced by the author (Clarke 1960a) to explain the much simpler behaviour in the relaxation zone behind a sharp corner in supersonic flow, namely that the outgoing pressure waves generated by the body are reflected back towards the surface by the relaxation-generated vorticity distribution (see Section 2 ). It is not surprising to find that the sigr.s and magnitudes of these reflected waves depend on body shape and Mach number regime. To conclude this section, we remark that past experience has indicated that a linear theory is capable of yielding quite accurate estimates of the superimposed effects of relaxation, even though the basic quantities (like (C.P.) for example) are not so well predicted. Also, it must be emphasised that the solutions discussed here and indeed in the Section to follow, are only valid on and near the body surface.

7. The Temperature Variations

In order to find the variations of the irternal mode and translational temperatures, we first note that the energy equation can be written in terms of the specific enthalpy, $h$, and pressure as fcllows (see equation 22),

$$
\rho \frac{\mathrm{Dh}}{\mathrm{D} t}-\frac{\mathrm{Dp}}{\mathrm{D} t}=0 \text {. }
$$

The enthalpy $h$ can be written as $\mathrm{C}_{\mathrm{p}_{4}} \mathrm{~T}_{1}+\mathrm{C}_{\mathrm{V}_{2}} \mathrm{~T}_{2}$ for present purposes and, consistent with the previous linearisations, $\rho$ in equation 22 can be replaced by its undisturbed stream value $\rho_{\infty}$. Then equation 22 can be 
integrated to give the approximate result

$$
\mathrm{C}_{\mathrm{p}_{1}} \Delta \mathrm{T}_{1}+\mathrm{C}_{\mathrm{V}_{2}} \Delta \mathrm{T}_{2} \simeq \rho_{\infty}^{-1}\left(\mathrm{p}-\mathrm{p}_{\infty}\right)
$$

We have written

$$
\Delta T_{1}=T_{1}-T_{1 \infty}: \Delta T_{2}=T_{2}-T_{1 \infty},
$$

since $T_{2 \infty}=T_{1 \infty}$. It follows from equations 8 and 96 that

$$
\tau \frac{D \Delta T_{2}}{D t}+\Delta T_{2} \simeq \frac{U^{2}(C \cdot P \cdot)}{2 C_{p}}
$$

Putting $\mathrm{D} / \mathrm{Dt} \simeq \mathrm{U} \partial / \partial \mathrm{x}$ as before we readily find that

$$
\Delta T_{2}=\frac{U}{2 \tau C_{p}} \int_{-\infty}^{x}\left(C \cdot P_{0}\right) e^{-(x-y) / \lambda} d y
$$

in subsonic flow. In supersonic flow the lower limit can be replaced by zero. Using equation 8 , and after some rearrangement, we find that

$$
\Delta T_{i}=\frac{U^{2}(C \cdot P .)}{2 C_{p}}+\left(\frac{C_{v_{2}}}{C_{p_{1}}}\right) \cdot \frac{U^{2}}{2 C_{p}} \int_{-\infty}^{x} \frac{\partial(C . P .) e^{-(x-y) / \lambda}}{\partial y} d y
$$

Integrating equation 99 by parts and combining the result with equation 100 shows that

$$
T_{1}-T_{2}=\frac{U^{2}}{2 C_{p_{1}}} \int_{-\infty}^{x} \frac{\partial(C \cdot P \cdot)}{\partial y} e^{-(x-y) / \lambda} d y
$$

This last result shows at once that an upstream influence of the relaxation effects exists for the temperatures in subsonic flow, since a (C.P.)/ $\partial \mathrm{y} \neq 0$ for $\mathrm{y}<0$ in that case.

It is important to observe that the temperatures on and near the body are functions of the whole pressure coefficient. In particular this fact is significant when we examine the difference between $T_{1}$ and $T_{2}$. In the 
singular case $\lambda=0$ the difference vanishes of course, but we may use the other limiting case of $\lambda \rightarrow \infty$ to illustrate the practical situation. For the near-frozen state we may write $T_{2} \simeq T_{1 \infty}$, whence $T_{1}-T_{2} \simeq \Delta T_{1}$ and we find that this difference in the limit is just

$$
\frac{\mathrm{U}^{2}}{2 \mathrm{C}_{\mathrm{p}_{1}}} \text { (C.P.), }
$$

(using either equation 100 or equation 101). Thus the difference between $T_{1}$ and $T_{2}$ depends on the body shape and attitude in the same way as (C.P.). Clearly this behaviour occurs for all non-zero $\lambda$ but we expect to find the amplitude of the difference decreasing in a general way with decreasing $\lambda$. It may be of some significance however that relatively large differences between $T_{1}$ and $T_{2}$ could arise locally due to some pecularity in body shape. The results presented above enable calculations of $T_{1}$ and $T_{2}$ to be made (within the confines of the slender body approximation) and these values could be used to estimate temperatures at the edge of the thermal boundary layer. The latter statement is made with reservations, since it may happen that the concept of the boundary layer and the "body-plus-displacement-thickness" treatment which is implied by it, begin to lose validity at pressures (and hence broadly speaking, Reynolds numbers) low enough to make $\lambda / L$ sufficiently large. (The question of relaxation efiects on such a flow is an interesting one, but outside our present scope. From the foregoing results we might expect that viscous and relaxation effects will be comparable over large regions of the flow field). Clearly any particular case must be examined on its merits, but we may quote the case of carbon dioxide, for which appreciable relaxation effects are present at pressures high enough for the low density aspects of viscous flow (such as slip phenomena) to be insignificant. In other words, the mean free molecular path will be small compared with the boundary layer thickness in this case. The 
non-dimensional group which roughly determines the state of the internal energy mode in a boundary layer is $\left(\delta_{1}^{2} / \tau^{\prime} /\right)^{\frac{1}{2}}$, where $\delta_{1}$ is boundary layer thickness and $X$ is the self diffusion coefficient for the particular (pure) gas in question. Assuming a laminar boundary layer, which seems reasonable in the circumstances, $\delta_{1} \sim \mathrm{x}^{\frac{1}{2}}(v / U)^{\frac{1}{2}}$, where $v$ is the kinematic viscosity. Thus

$$
\left(\frac{\delta^{2}}{\tau^{\prime} \Theta}\right)^{\frac{1}{2}} \sim\left(\frac{\mathrm{x}}{\mathrm{L}}\right)^{\frac{1}{2}}\left(\frac{\mathrm{L}}{\tau^{\prime} \mathrm{U}}\right)^{\frac{1}{2}}\left(\frac{v}{\delta}\right)^{\frac{1}{2}} \text {. }
$$

The ratio $v / g \sim 1$ for a number of gases and it follows that if the external (inviscid) flow is near-frozen it will also be near-frozen in the boundary layer. The appearance of the ratio $x / L$ above guarantees that it will he frozen at the nose of the body but it is significant to notice that it is $\left(L / \tau^{\prime} U\right)^{\frac{1}{2}}$ for coughly, $\lambda^{-\frac{1}{2}}$ ) which determines boundary layer behaviour whilst that in the external flow is governed by the value $\lambda^{-1}$.

In cases where near frozen flow occurs in the layer, the effectiveness of the actual material of the body's surface in accommodating the internal mode becomes important in the determination of energy flux rates, (see for example, Clarke, 1960b). Thus the interpretation of heat transfer measurements on bodies in polyatomic gas flows must be approached with caution. Similar arguments apply with even greater force to the case of chemically reacting gas flows, on account of the greater energies involved and the possibility of finding wide ranges of catalytic efficiencies amongst practical materials. Remarks such as these might apply to heat transfer measurements on, let us say, a slender cone in a typical shock tube. Results like those presented above may be used to estimate the conditions at the edge of the boundary layer in such a case.

The author would like to acknowledge the helpful advice and criticism received from Professor G. N, Ward and Mr. G. M. Lilley during the preparation of this paper. 
8. References

1. Chu, B.T. 1957 Wave propagation and the method of characteristics in reacting gas mixtures with applications to hypersonic flow. WADC TN. 57-213

2. Clarke, J.F. 1958 The flow of chemically reacting gas mixtures. College of Aeronautics Report 117.

1960a The linearised flow of a dissociating gas.

Jnl. Fluid Mech. vol. 7, pp 577-595.

$1960 \mathrm{~b}$ Heat conduction through a gas with one inert internal mode. College of Aeronautics Note 102.

3. Erdelyi, A. 1953 Figher Transcendental Functions, vol. Il. Magnus, W. McGraw Hill Book Co. Inc., New York. Oberhettinger, F. Tricomi, F.G.

4. Gunn, J.C. 1952 Relaxation time effects in gas dynamics. Aero. Res. Council, London, R\&M 2338.

5. Kirkwood, J.G. 1957 Hydrodynamics of a reacting and Wood, W. W. relaxing fluid. Jnl. App. Physics vol. 28, pp 395-398.

6. Lighthill, M.J. 1945 Sup€soric flow past bodies of revolution. Aero. Res. Council, London, R\&M 2003

7. Moore, F.K. 1960 Propagation of weak disturbances in a Gibson, W.E. gas subject to relaxation effects. Jnl. Aero/Space Sciences, vol. 27 p.117

8. Sears, W.R. 1953 Slender-body theory - Review and Adams, M. C. extension. Jnl. Aero. Sciences, vol. 20, pp 85-98. 


\section{References (Continued)}

9. Vincenti, W.G. 1959 Non-equilibrium flow over a wavy wall. Dept. of Aero. Eng., Stanford University SUDAER No. 85.

10. Ward, G.N. 1949 Supersonic flow past slender pointed bodies.

Quarterly Jnl. Mech. \& App. Maths., vol.2, pp 75-97. 
APPENDIX A

Inversion Integrals for the Function $b_{0}(x)$

Case (i)

In the subsonic case $b_{0}(x)$ is written as

$$
\mathrm{b}_{\mathrm{o}}(\mathrm{x})=-\mathrm{A}_{\mathrm{o}}(\omega)\left\{\mathrm{C}+\log \left(\beta_{\mathrm{f}}|\omega| \bar{z} / 2\right)\right\},
$$

(see equations 35,36 and 45). To accomplish the inversion integration we write

$$
\begin{aligned}
& b_{o}(x)=-A_{o}(\omega) \log \left(\beta_{f} / 2\right)-i \omega\left(-A_{o}\right)\left[\frac{C+\log |\omega|}{-i \omega}\right] \\
& +\frac{1}{2}\left(-i \omega+\beta^{2} / \lambda\right)\left(-A_{o}\right)\left\{\frac{C+\log \left(\omega+i \beta^{2} / \lambda\right)}{-i\left(\omega+i \beta^{2} / \lambda\right)}\right\} \\
& -\frac{1}{2}(-i \omega+1 / \lambda)\left(-A_{o}\right)\left[\frac{C+\log (\omega+i / \lambda)}{-i(\omega+i / \lambda)}\right]
\end{aligned}
$$

The first term in equation $A .2$ is simply $a_{o}(x) \log \left(\beta_{f} / 2\right)$. To invert the remainder we make use of the fact that $-i \omega$ is equivalent to the operation $\partial / \partial x$ and treat the remaining parts by means of the Falting theorem for complex Fourier transforms, which states that

$$
\frac{1}{\sqrt{2 \pi}} \int_{-\infty}^{\infty} f(y) g(x-y) d y=F(\omega) G(\omega) \text {. }
$$

$F$ and $G$ are the transforms of $f(x)$ and $g(x)$. Thus we can deal with the ' $\log ^{\prime}$ terms in curly brackets separately, taking care to use the contour described in Section 3, Case (i).

The first curly bracket term in equation $\mathrm{A} .2$ inverts to a function $g_{1}(x)$ where

$$
\begin{aligned}
& \sqrt{2 \pi} g_{1}(x)=\operatorname{Lt}_{\epsilon \rightarrow 0}\left\{\int_{\epsilon}^{\infty}(C+\log u) e^{i x u} \frac{d u}{i u}-\int_{\epsilon}^{\infty}(C+\log u) e^{-i x u} \frac{d u}{i u}\right\} \\
& =\frac{|x|}{x} \operatorname{Lt}_{\epsilon \rightarrow o}\left\{\int_{\epsilon}^{\infty}(C+\log u) e^{i|x| u} \frac{d u}{i u}-\int_{\epsilon}^{\infty}(C+\log u) e^{-i|x| u} \frac{d u}{i u}\right\}
\end{aligned}
$$


After some manipulation it follows that

$$
g_{1}(x)=-\sqrt{\frac{\pi}{2}} \frac{|x|}{x} \log |x|
$$

In dealing with the second and third curly bracket terms in eqation A. 2, which we call $g_{2}(x)$ and $g_{3}(x)$ in the physical plane, respectively, the complex $\omega-$ plane can be cut from $-\infty-i \beta^{2} / \lambda$ to $-i \beta^{2} / \lambda$ and $-\infty-i / \lambda$ to $-i / \lambda$ in each case and the real $\omega$-axis contour shifted down to positions just above each branch cut, indented above the singularity, and running on to $+\infty-i \beta^{2} / \lambda$ and $+\infty-i / \lambda$ respectively. Taking $g_{2}(x)$, we find that

$$
\sqrt{2 \pi} e^{\beta^{2} x / \lambda} g_{2}(x)=\int_{-\infty}^{\infty}(C+\log z) e^{-i x z} \frac{d z}{-i z}
$$

where $\mathrm{z}$ is a new variable related to $\omega$ by

$$
z=\omega+i \beta^{2} / \lambda
$$

It follows from equation A. 5 that $g_{2}(x)=0$ for $x<0$. When $x>0$,

$$
\begin{aligned}
\sqrt{2 \pi} \mathrm{e}^{\beta^{2} \mathrm{x} / \lambda} \mathrm{g}_{2}(\mathrm{x})= & \int_{\varepsilon}^{\infty}(\mathrm{C}+\log \xi+i \pi) \mathrm{e}^{i x \xi} \frac{\mathrm{d} \xi}{i \xi}+\int_{0}^{\pi}\left(\mathrm{C}+\log \epsilon \mathrm{e}^{i \theta}\right) \mathrm{e}^{-i \mathrm{x} \epsilon \mathrm{e}^{i \theta}} \mathrm{d} \theta \\
& -\int_{\epsilon}^{\infty}(\mathrm{C}+\log \xi) \mathrm{e}^{-i \mathrm{x} \xi} \frac{\mathrm{d} \xi}{\mathrm{i} \xi},
\end{aligned}
$$

in the limit as $\epsilon \rightarrow 0$. ( $z$ is put equal to $\xi$ on the real $z$ axis and equal to $\epsilon \mathrm{e}^{i \theta}$ on the indentation around the branch point). The integrals in equation $A .7$ can be rearranged and it is then possible to show that

$$
\begin{aligned}
g_{2}(x) & =-\sqrt{2 \pi} e^{-\beta^{2} x / \lambda} \log x+i \pi^{2} \frac{e^{-\beta^{2} x / \lambda}}{\sqrt{2 \pi}} ; x>0 \\
& =0 ; \quad x<0
\end{aligned}
$$

The value for $g_{3}(x)$ follows at once on setting $\beta^{2}$ in equation $A .8$ equal to 1 .

The function $f(y)$ in equation $A .3$ we can identify as $a_{0}(y)$ in tile notation of the text, whence using the theorem expressed there together with the results derived above it follows that 


$$
\begin{aligned}
b_{0}(x) & =a_{0}(x) \log \left(\beta_{f} / 2\right)-\frac{1}{2} \frac{\partial}{\partial x} \int_{-\infty}^{x} a_{0}(y) \log (x-y) d y \\
+ & \frac{1}{2} \frac{\partial}{\partial x} \int_{x}^{\infty} a_{0}(y) \log (y-x) d y-\frac{1}{2}\left(\frac{\partial}{\partial x}+\frac{\beta^{2}}{\lambda}\right) \int_{-\infty}^{x} a_{0}(y) e^{-\beta^{2}(x-y) / \lambda_{l}} \log (x-y) d y \\
& +\frac{1}{2}\left(\frac{\partial}{\partial x}+\frac{1}{\lambda}\right) \int_{-\infty}^{x} a_{0}(y) e^{-(x-y) / \lambda} \log (x-y) d y
\end{aligned}
$$

The imaginery terms in $g_{2}(x)$ and $g_{3}(x)$ are found to cancel out in the expression for $b_{0}(x)$, as indeed they must. If the indicated operations are performed on the integrals in equation $A .9$ and the value $U^{\prime}(x) / 2 \pi$ substituted for $a_{0}(x)$ the result quoted in equation 49 is obtained. We note that $a_{0}(x)=0$ for $x<0$ and $x>L$; whence the limits in equation 49 follow.

\section{Case (ii)}

In the transonic case (see Section 3, Case (ii)) we write the transform of $b_{0}(x)$ in the same way as in equation A. 2 with the following differences. $|\omega|$ is replaced by $\omega, \beta^{2}$ is replaced by $-\bar{\beta}^{2}$ and $Z$ is replaced by $Z^{\prime}$. Inversior now takes place just above the real $\omega$-axis, indented to pass above the point $\omega=0$. These differences have the following effects. Using a notation consistent with that of the previous case,

$$
\begin{aligned}
g_{1}(x) & =-\sqrt{2 \pi} \log x+\frac{i \pi^{2}}{\sqrt{2 \pi}} ; x>0 \\
& =0 ; x<0 .
\end{aligned}
$$

Because having $-\bar{\beta}^{2}$ for $\beta^{2}$ in the $g_{2}$ term puts the relevant singularity above the real $w$-axis, it follows that

$$
\begin{aligned}
g_{2}(x) & =0 ; x>0 \\
& =\sqrt{2 \pi} \quad e^{-\beta^{2}|x| / \lambda} \log |x| ; x<0
\end{aligned}
$$

The function $g_{3}(x)$ is unchanged (see equation A. 8 with $\beta^{2}=1$ ). Using the theorem in equation A. 3 etc., it follows that 


$$
\begin{aligned}
b_{0}(x) & =a_{0}(x) \log \left(\beta_{\hat{f}} / 2\right)-\frac{\partial}{\partial x} \int_{-\infty}^{x} a_{0}(y) \log (x-y) d y \\
& +\frac{1}{2}\left(\frac{\partial}{\partial x}-\frac{\bar{\beta}^{2}}{\lambda}\right) \int_{x}^{\infty} a_{c}(y) e^{-\bar{\beta}^{2}(y-x) / \lambda} \log (y-x) d y \\
& +\frac{1}{2}\left(\frac{\partial}{\partial x}+\frac{1}{\lambda}\right) \int_{-\infty}^{x} a_{0}(y) e^{-(x-y) / \lambda} \log (x-y) d y
\end{aligned}
$$

the imaginery parts of the $g(x)$ functions cancelling out as before. With a little rearrangement and the substitution of the value for $a_{0}(x)$ we easily obtain equation 56 , provided that we let $\mathrm{y}$ approach $\mathrm{x}$ in the last two integrals there like $\mathrm{x}+\epsilon$ and $\mathrm{x}-\epsilon$ respectively(i.e. take a "principal value").

\section{Case (iii)}

Once again we use a form of $b_{o}(x)$ like that in equation $A .2$ only here we write $B_{f}$ for $\beta_{f}, B^{2}$ for $\beta^{2}, i \omega$ for $|\omega|$ and $Z^{\prime \prime}$ for $Z$ in the first curly bracket expression.

It follows that the $g_{2}(x)$ and $g_{3}(x)$ terms are identical with those of Case (i) when $B^{2}$ is written for $\beta^{2}$ and using the contour described in Section 3, Case (iii) for the "log(iw)" term we find that

$$
\begin{aligned}
g_{1}(x) & =-\sqrt{2 \pi} \log x ; x>0 \\
& =0 ; x<0
\end{aligned}
$$

The result in equation 62 now follows in the usual way. We remark here that the Laplace transform could easily be used in the fully supersonic case, with its absence of upstream influence effects. The Fourier transform treatment has been retained since the problem can then be dealt with in a unified fashion. 


\section{APPENDIX B}

Evaluation of the Relaxation Drag Integral

The relaxation drag integral can be written generally in the form

$2 \pi S_{r} C_{D_{r}}=\int_{0}^{I} S^{\prime \prime}(x) \int_{0}^{x} S^{\prime \prime}(y)\left\{E i\left(\frac{y-x}{\lambda}\right)-E i\left(\alpha^{2} \frac{y-x}{\lambda}\right)\right\} d y d x$, ....

where $\alpha^{2}$ may be $\beta^{2}, \bar{\beta}^{2}$ or $B^{2}$ depending on the Mach number regime. The inner integral in equation $B .1$, is equal to $2 I_{\alpha}-2 S^{\prime}(x) \log \alpha$ in the notation of equation 50 et seq.

Writing

$$
\mathrm{x}-\mathrm{y}=\lambda \sigma
$$

this inner integral can be rewritten in the form

$$
2 I_{\alpha}-2 S^{\prime}(x) \log \alpha=\lambda \int_{0}^{x / \lambda} S^{\prime \prime}(x-\lambda \sigma)\left\{E i(-\sigma)-\operatorname{Ei}\left(-\alpha^{2} \sigma\right)\right] d \sigma \text {. }
$$

The cross-section area $S(x)$ of a wide variety of body shapes can be expressed as a polynomial in $\mathrm{x}$, whence it follows from equation $\mathrm{B} .3$, that we shall have to deal with a number of integrals of the type

$$
I_{n}=\int_{0}^{x / \lambda} \sigma^{n}\left\{E_{1}\left(\alpha^{2} \sigma\right)-E_{1}(\sigma)\right\} d \sigma
$$

In writing equation $B .4$, use has been made of the rather more convenient notation for the exponential integral which puts

$$
\mathrm{E}_{1}(\sigma)=-\operatorname{Ei}(-\sigma)
$$

etc. (See Erdelyi, et al, 1953). Then, using the results given in this reference, it is found that

$$
\begin{aligned}
(n+1) I_{n} & =\Gamma(1+n)\left[\alpha^{-2(n+1)}-1\right]-\alpha^{-2(n+1)}\left[\Gamma\left(1+n, \alpha^{2} x / \lambda\right)-\left(\alpha^{2} x / \lambda\right)^{n+1} E_{1}\left(\alpha^{2} x / \lambda\right)\right] \\
& +\Gamma(1+n, x / \lambda)-(x / \lambda)^{n+1} E_{1}(x / \lambda)
\end{aligned}
$$

The functions $I(1+n, x / \lambda)$ etc. in equation B. 6 are one form of the incomplete gamma function, 


$$
\Gamma(1+n, x / \lambda)=\int_{x / \lambda}^{\infty} e^{-t} t^{n} d t
$$

It follows that $I_{\alpha}$ can now be expressed in terms of a sum involving the appropriate $I_{n}$ 's with suitable coefficients. The restriction to pointed nosed bodies ensures that the body radius $R(x)$ will behave like $x$, at worst, in the nose region. Hence $\mathrm{n}$ in equation B. 4 will never be less than zero.

In evaluating the drag integral in equation B. 1 we now encounter terms like

$$
\begin{aligned}
& J_{n, a}=\int_{0}^{1} x^{n}\left\{\Gamma(a, x / \lambda)-\alpha^{-2 a} \Gamma\left(a, \alpha^{2} x / \lambda\right)\right\} d x, \\
& K_{n}=\int_{0}^{1} x^{n}\left\{E_{1}\left(\alpha^{2} x / \lambda\right)-E_{1}(x / \lambda)\right\} d x .
\end{aligned}
$$

The body length $L$ has been set equal to 1 here. There is no loss of generality in so doing but all lengths are hereafter measured in terms of $L$ as the basic dimension. In other words $\lambda$ in equations B. 8 and B. 9 (and subsequently) stands for the ratio of relaxation length to body length. We find that

$$
\begin{aligned}
(n+1) J_{n, a} & =\Gamma(a)-y(a, 1 / \lambda)+\lambda^{n+1} y(a+n+1,1 / \lambda)-\alpha^{-2 a} \Gamma(a) \\
& +\alpha^{-2 a} y\left(a, \alpha^{2} / \lambda\right)-\alpha^{-2(a+n+1)} \lambda^{n+1} y\left(a+n+1, \alpha^{2} / \lambda\right),
\end{aligned}
$$

where $y(a, 1 / \lambda)$ etc. stands for the other incomplete gamma function,

Also

$$
y(a, 1 / \lambda)=\int_{0}^{1 /} e^{-t} t^{a-1} d t \text {. }
$$

$$
\begin{aligned}
(\mathrm{n}+1) K_{n} & =\alpha^{-2(n+1)} \lambda^{n+1} \gamma\left(n+1, \alpha^{2} / \lambda\right)+E_{1}\left(\alpha^{2} / \lambda\right) \\
& -\lambda^{n+1} y(n+1,1 / \lambda)-E_{1}(1 / \lambda) .
\end{aligned}
$$


Some results have been computed for the parabolic arc meridian profile body, for which

$$
P(x)=4 \delta\left(x-x^{2}\right) \text {. }
$$

This has

$$
S^{\prime \prime}(x)=32 \pi \delta^{2}\left(1-6 x+6 x^{2}\right),
$$

whence it follows from the general results given above that

$$
\begin{aligned}
& 2(32 \delta)^{-2} C_{D r}=\frac{1}{5}\left[\frac{\lambda}{\alpha^{2}}\left(1-e^{-\alpha^{2} / \lambda}\right)-\lambda\left(1-e^{-1 / \lambda}\right)\right] \\
& -\frac{1}{2}\left[\left(\frac{\lambda}{\alpha^{2}}\right)^{2}\left(1-e^{-\alpha^{2} / \lambda} e_{1}\left(\alpha^{2} / \lambda\right)\right)-\lambda^{2}\left(1-e^{-1 / \lambda} e_{1}(1 / \lambda)\right)\right] \\
& +3\left[\left(\frac{\lambda}{\alpha^{2}}\right)^{4}\left(1-e^{-\alpha^{2} / \lambda} e_{3}\left(\alpha^{2} / \lambda\right)\right)-\lambda^{4}\left(1-e^{-1 / \lambda} e_{3}(1 / \lambda)\right)\right] \\
& -24\left[\left(\frac{\lambda}{\alpha^{2}}\right)^{6}\left(1-e^{-\alpha^{2} / \lambda} e_{5}\left(\alpha^{2} / \lambda\right)\right)-\lambda^{6}\left(1-e^{-1 / \lambda} e_{5}(1 / \lambda)\right)\right],
\end{aligned}
$$

provided that we take $S_{r}=\pi \delta^{2}$.

The functions $e_{1}, e_{3}$ and $e_{5}$ appearing in equation $B .15$ are the truncated exponential series

$$
e_{n}(z)=\sum_{m=0}^{n} \frac{z^{m}}{m !} .
$$

They arise from the fact that when $\mathrm{n}$ is an integer the incomplete gamma function $y(1+n, z)$ can be expressed in the form

$$
y(1+n, z)=n !\left[1-e^{-z} e_{n}(z)\right] \text {. }
$$




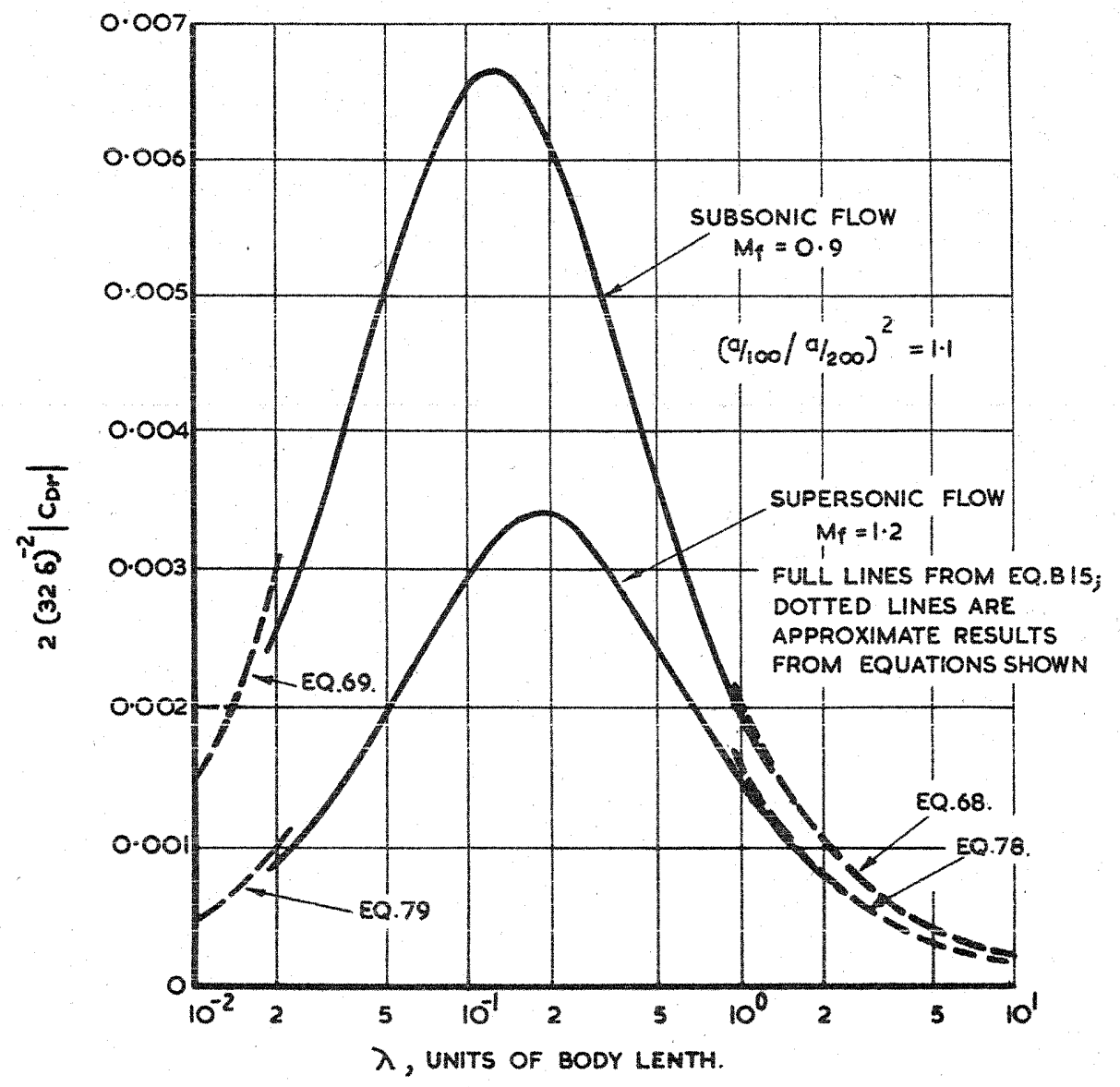

FIG.I. RELAXATION DRAG FOR A PARABOLIC ARC BODY

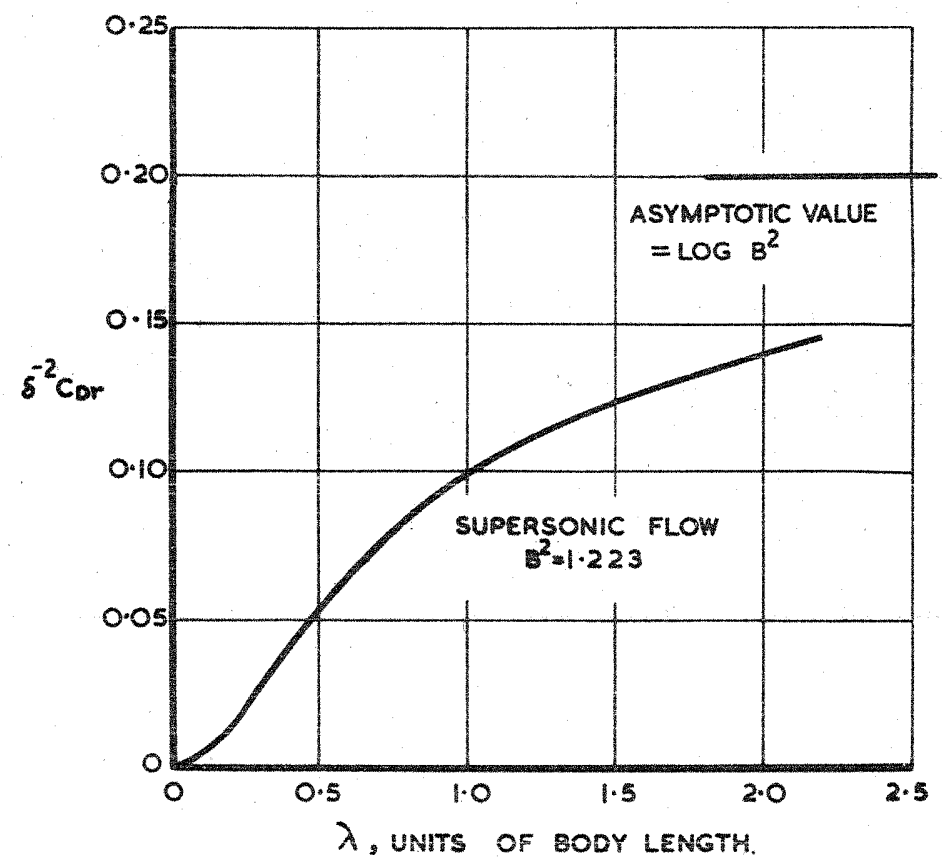

FIG.2. RELAXATION DRAG FOR A CONE 